\title{
Hinged Dissections Exist
}

\author{
Timothy G. Abbott • Zachary Abel • \\ David Charlton • Erik D. Demaine • \\ Martin L. Demaine • Scott Duke Kominers
}

Received: 2 September 2008 / Revised: 16 May 2010 / Accepted: 23 June 2010 /

Published online: 1 October 2011

(C) Springer Science+Business Media, LLC 2011

\begin{abstract}
We prove that any finite collection of polygons of equal area has a common hinged dissection. That is, for any such collection of polygons there exists a chain of polygons hinged at vertices that can be folded in the plane continuously
\end{abstract}

T.G. Abbott's research was partially supported by an NSF Graduate Research Fellowship and an MIT-Akamai Presidential Fellowship.

E.D. Demaine's research was partially supported by NSF CAREER award CCF-0347776, DOE grant DE-FG02-04ER25647, and AFOSR grant FA9550-07-1-0538.

S.D. Kominers' research was partially supported by an NSF Graduate Research Fellowship.

T.G. Abbott · E.D. Demaine · M.L. Demaine

MIT Computer Science and Artificial Intelligence Laboratory, 32 Vassar Street, Cambridge, MA 02139, USA

T.G. Abbott

e-mail: tabbott@mit.edu

E.D. Demaine

e-mail: edemaine@mit.edu

M.L. Demaine

e-mail: mdemaine@mit.edu

\section{Z. Abel (凶)}

Department of Mathematics, Massachusetts Institute of Technology, 77 Massachusetts Avenue, Cambridge, MA 02139, USA

e-mail: zabel@math.mit.edu

D. Charlton

Department of Computer Science, Boston University, 111 Cummington Street, Boston, MA 02215, USA

e-mail: dchar@mit.edu

S.D. Kominers

Department of Economics, Harvard University, and Harvard Business School, Wyss Hall, Soldiers Field, Boston, MA 02163, USA

e-mail: kominers@fas.harvard.edu 
without self-intersection to form any polygon in the collection. This result settles the open problem about the existence of hinged dissections between pairs of polygons that goes back implicitly to 1864 and has been studied extensively in the past ten years. Our result generalizes and indeed builds upon the result from 1814 that polygons have common dissections (without hinges). Our proofs are constructive, giving explicit algorithms in all cases. For two planar polygons whose vertices lie on a rational grid, both the number of pieces and the running time required by our construction are pseudopolynomial. This bound is the best possible, even for unhinged dissections. Hinged dissections have possible applications to reconfigurable robotics, programmable matter, and nanomanufacturing.

Keywords Folding · Reconfiguration · Hinge · Polygon · Refinement

\section{Introduction}

Around 1808, Wallace asked whether every two polygons of the same area have a common dissection, that is, whether any two equal-area polygons can be cut into a finite set of congruent polygonal pieces [19, p. 222]. Figure 1 shows a simple example. Lowry [31] published the first solution to Wallace's problem in 1814, although Wallace may have also had a solution at the time; he published one in 1831 [40]. Shortly thereafter, Bolyai [5] and Gerwien [21] rediscovered the result, causing this result to be known sometimes as the Bolyai-Gerwien Theorem.

Lowry's dissection construction, as described by Frederickson [19], is particularly elegant and uses a pseudopolynomial number of pieces. In this paper, pseudopolynomial means polynomial in the combinatorial complexity $(n)$ and the dimensions of an integer grid on which the input is drawn. Although Lowry's construction does not require the vertices to have rational coordinates, pseudopolynomial is easiest to define in this case. ${ }^{1}$ A pseudopolynomial bound is the best possible in the worst case: dissecting a polygon of diameter $x>1$ into a polygon of diameter 1 (for example, a long skinny triangle into an equilateral triangle) requires at least $\lceil x\rceil$ pieces.

With this worst-case result in hand, attention has turned to optimal dissections using the fewest pieces possible for the two given polygons. This problem has been studied extensively for centuries in the mathematics literature [8, 19, 35] and the puzzle literature [29, 30, 32, 36], and more recently in the computational geometry literature [3, 9, 27].

Hinged dissections are dissections with an additional constraint: the polygonal pieces must be hinged together at vertices into a connected assembly. The first published hinged dissection appeared in 1864, illustrating Euclid's Proposition I.47 [26]; see [20, pp. 4-5]. The most famous hinged dissection is Dudeney's 1902 hinged dissection [16]; see Fig. 2. This surprising construction inspired many to investigate hinged dissections; see, for example, Frederickson's book on the topic [20].

\footnotetext{
${ }^{1}$ A general definition of pseudopolynomial (beyond the rational case) is polynomial in terms of $n$ and a geometric ratio such as the largest distance in the input polygons divided by the smallest distance between nonincident edges. Such bounds are beyond the scope of this paper; even for Lowry's construction, it would require care and has not been previously attempted.
} 
Fig. 1 4-piece dissection of Greek cross to square from 1890 [29]
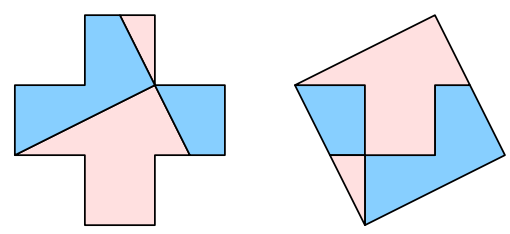
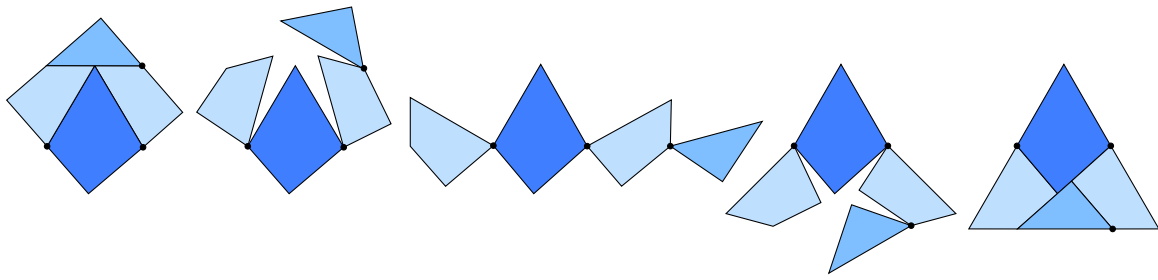

Fig. 2 Dudeney's 1902 hinged dissection of a square into a triangle [16]

However, the fundamental problem of general hinged dissection has remained open [13, 34]: do every two polygons of the same area have a common hinged dissection? This problem has been attacked in the computational geometry literature [2, $14,15,18]$ but has only been solved in special cases. For example, all polygons made from edge-to-edge gluings of $n$ identical subpolygons (such as polyominoes) have been shown to have a common hinged dissection [14]. Perhaps most intriguingly, Eppstein [18] showed that the problem of finding a common hinged dissection of any two triangles of equal area is just as hard as the general problem.

Hinged dissections are particularly exciting from the perspectives of reconfigurable robotics, programmable matter, and nanomanufacturing. Recent progress has enabled chemists to build millimeter-scale "self-working" 2D hinged dissections such as Dudeney's [33]. An analog for 3D hinged dissections may enable the building of a complex 3D structure out of a chain of units; see [22] for one such approach. We could even envision an object that can re-assemble itself into different $3 \mathrm{D}$ structures on demand [15]. This approach contrasts existing approaches to reconfigurable robotics (see, for example, [38]), where units must reconfigure by attaching and detaching from each other through a complicated mechanism.

Our Results We settle the hinged dissection open problem, first formally posed in 1999 [14] but implicit back to 1864 [26] and 1902 [16]. Specifically, Sect. 3 proves a universality result: any two polygons of the same area have a common hinged dissection. In fact, our result is stronger, building a single hinged dissection that can fold into any finite set of desired polygons of the same area. The analogous multipolygon result for (unhinged) dissections is obvious - simply overlay the pairwise dissections - but no such general combination technique is known for hinged dissections. Indeed, the lack of such a transitivity construction has been the main challenge in constructing general hinged dissections.

Our construction starts from an arbitrary (unhinged) dissection, such as Lowry's [31]. We show that any dissection of a finite set of polygons can be subdivided and hinged so that the resulting hinged dissection folds into all of the original polygons. 
We give a method of subdividing pieces of a hinged figure that effectively allows us to "unhinge" a portion of the figure and "re-attach" it at an alternate location. This construction allows us to "move" pieces and hinges around arbitrarily, at the cost of extra pieces. In this way, we show how to hinge any dissection.

This initial construction may easily require an exponential number of pieces. However, we show in Sect. 5 that a more careful execution of Lowry's dissection [31] attains a pseudopolynomial number of pieces for two target polygons when the input vertices lie on a rational grid. As mentioned above, such a bound is essentially best possible, even for unhinged dissections (although we likely do not obtain the optimal constant exponent). This more efficient construction requires substantially more complex gadgets for simultaneously moving several groups of pieces at roughly the same cost as moving a single piece, and relies on specific properties of Lowry's dissection. The more general problem of efficient hinge dissection when input coordinates may be irrational (or rational, but with denominators having an intractably large least common multiple) remains open.

We also solve another open problem concerning the precise model of hinged dissections. In perhaps the most natural model of hinged dissections, pieces cannot properly overlap during the folding motion from one configuration to another. However, all theoretical work concerning hinged dissections $[2,14,15,18]$ has only been able to analyze the "wobbly hinged" model [20], where pieces may intersect during the motion. Is there a difference between these two models? Again this problem was first formally posed in 1999 [14]. We prove in Sect. 4 that any wobbly hinged dissection can be subdivided to enable continuous motions without piece intersection, at the cost of increasing the combinatorial complexity of the hinged dissection by only a constant factor. This result builds on the Carpenter's Rule Theorem [6], the theory of slender adornments [7], and a recent extension to self-touching linkages [1].

The following theorem summarizes our results:

Theorem 1 Any finite set of polygons of equal area have a common hinged dissection that can fold continuously without intersection between the polygons. For two target polygons with vertices drawn on a rational grid, the number of required pieces is pseudopolynomial, as is the running time of the algorithm to compute the common hinged dissection.

One interesting consequence of this theorem is that any finite set of polyhedral surfaces of equal surface area have a common hinged dissection: It is known that every polyhedral surface can be triangulated and then vertex-unfolded into a hinged chain of triangles [12]. Our results (specifically Theorem 6) show how to construct a single hinged chain that can fold into any finite set of such chains, which can then be folded (and glued) into the polyhedral surfaces.

\section{Terminology}

In this section, we introduce, formalize, and motivate the key terminology used throughout the paper. The two central concepts are "hinged figures" and "refinements", which together embody a generalized notion of "hinged dissection". An intuitive definition of hinged dissections is clear: cut up one shape, hinge the pieces 
Fig. 3 A tree-like hinged figure, its incidence graph (bold), and part of its boundary path

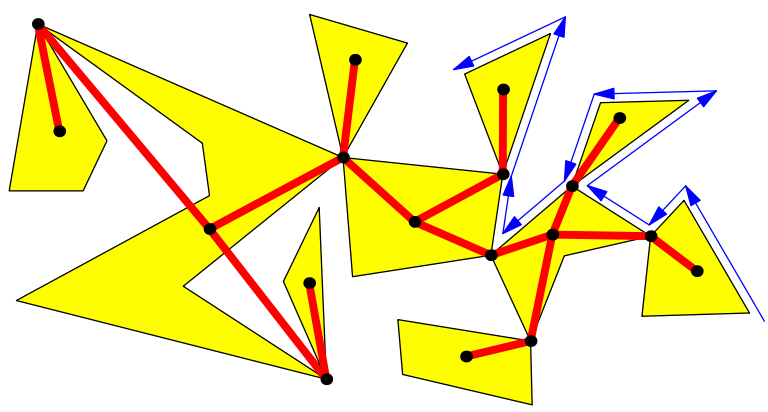

together, fold, and glue back together into another shape. But defining cuts, gluing, and valid folding require care and these notions can be difficult to work with, so we take a somewhat different approach. We define a "hinged figure" to be a generalized abstract form of hinged dissection - pieces hinged together-which can have many different configurations or possibly none at all. We call one hinged figure a "refinement" of another if the former can take on all the shapes of the latter, intuitively because the former comes from cutting up and hinging the latter. In particular, these notions give us a definition for hinged dissection: a hinged figure that is simultaneously a refinement of two or more given polygons. But the notion of refinement is much more powerful than this, because it allows refining general hinged figures and not just polygons, and this idea will be central to our construction.

\subsection{Hinged Figures}

A hinged figure A (see Fig. 3) is defined formally as a finite collection of simple, oriented polygons (the pieces of $A$ ) together with a finite collection of hinges, where a hinge is a (nonempty) finite cyclic list of vertices of pieces. These pieces and hinges must satisfy the following conditions:

1. The abstract topological space $\mathcal{T}(A)$, formed from the pieces of $A$ by identifying all vertices in each hinge of $A$, is connected.

2. Each hinge of $A$ contains at most one vertex from each piece of $A$.

3. Each vertex of each piece of $A$ is contained in at most one hinge. ${ }^{2}$

Intuitively, Condition 1 says that the pieces are joined into a connected assembly, Condition 2 says that no two vertices of any piece are "pinched" together, and Condition 3 guarantees that no two hinges are "collocated". The cyclic order of vertices defining hinge will indicate the counterclockwise order in which these pieces appear in the plane; this is formalized in the discussion of planar configurations below.

The incidence graph of a hinged figure is the bipartite graph that has a vertex corresponding to every piece and every hinge, such that two nodes are connected by an edge if one represents a piece and the other represents a hinge on that piece; see

\footnotetext{
${ }^{2}$ Technical comment: we consider two hinged figures to be equivalent if they differ only in the addition or removal of $180^{\circ}$ vertices of pieces and/or hinges incident with only one vertex.
} 
Fig. 3. Because $\mathcal{T}(A)$ is connected, $A$ 's incidence graph is also connected. ${ }^{3}$ A hinged figure is tree-like if the incidence graph is a tree, and it is chain-like if the incidence graph is an open chain, i.e. a path.

We do not require hinged figures to be tree-like in general, so that our definition captures many existing hinged dissections that hinge pieces together in cycles, such as Lindgren's classical dissection of two quadrilaterals with equal corresponding angles [19, p. 26] and the polyform hinged dissections of [14]. Nonetheless, most of the hinged figures generated by our algorithms will be tree-like.

A (planar) configuration of a hinged figure $A$ is a map $f: \mathcal{T}(A) \rightarrow \mathbb{R}^{2}$ satisfying the properties listed below. (Note that each piece of $A$ is naturally a subspace of $\mathcal{T}(A)$, so we may consider $f$ as a map from each piece separately such that all vertices in each hinge map to the same point in $\mathbb{R}^{2}$.)

1. Each piece is mapped by $f$ isometrically into the plane while preserving its orientation.

2. The images under $f$ of the interiors of distinct pieces do not intersect in the plane.

3 . For each hinge $h$, the cyclic order of pieces defining $h$ corresponds to the counterclockwise order of the images of those pieces around point $f(h)$ in the plane.

4. Whenever two distinct hinges map to the same point in the plane, the pieces incident to these hinges do not form a topological crossing at the point. (For two such hinges $h$ and $h^{\prime}$, two pieces $P$ and $Q$ incident to $h$ are topologically crossed with two pieces $P^{\prime}$ and $Q^{\prime}$ incident to $h^{\prime}$ if the counterclockwise cyclic order of the four polygons $f(P), f(Q), f\left(P^{\prime}\right), f\left(Q^{\prime}\right)$ in the plane around the point $f(h)=f\left(h^{\prime}\right)$ is $f(P), f\left(P^{\prime}\right), f(Q), f\left(Q^{\prime}\right)$ or $f(P), f\left(Q^{\prime}\right), f(Q), f\left(P^{\prime}\right)$.)

A hinged figure is realizable if it admits at least one such configuration.

Recall that our definition of hinged figure is primarily combinatorial. In particular, there is no stipulation that hinged figures be realizable. (A simple example of an unrealizable hinged figure is five squares joined at a common hinge.) The reason for our general definition is that some of our results, such as Lemma 4 below, make use of potentially unrealizable hinged figures as intermediate steps toward realizable hinged figures.

The boundary $\partial A$ of a hinged figure $A$ is the collection of oriented cycles formed from traversing all edges of all pieces in Euler-tour order, as illustrated in Fig. 3. The Euler-tour order is defined as follows: from a counterclockwise-oriented edge $u v$ of a piece $P$, we proceed to the counterclockwise-next edge $v w$ of $P$ if $v$ is not a hinge, or else we proceed to the counterclockwise-next edge $v w$ of the next piece $Q$ incident to the hinge $v$ (in other words, the next piece $Q$ appearing in the hinge's cyclic list). The boundary traces each hinge point multiple times; we distinguish these as distinct boundary points. For a tree- and chain-like hinged figure, the boundary consists of a single cycle incorporating all edges of the pieces.

It will be helpful to consider portions of hinged figures, called "subfigures". Suppose we have a hinged figure $A$ and a subset $S$ of $A$ 's pieces. Define the restriction of $A$ to $S$ to be the pieces of $S$ together with hinges defined as follows: for each hinge

\footnotetext{
${ }^{3}$ In particular, this fact follows because the incidence graph may be realized as a deformation retraction [24, p. 2] of $\mathcal{T}(A)$.
} 
$h$ of $A$ incident to at least one polygon in $S$, form the cyclic sublist of the vertices of $h$ belonging to pieces in $S$. The result is not necessarily connected, but instead forms a collection of disconnected hinged figures. This definition may be compared to the notion of "induced subgraph" from graph theory; in fact, the effect of the above restriction on the incidence graph of $A$ is to form the induced subgraph on the nodes corresponding to pieces in $S$ and hinges incident to something in $S$. We call a hinged figure $B$ a subfigure of $A$ if the pieces of $B$ are a subset of the pieces of $A$ and the restriction of $A$ to this subset of pieces is exactly $B$.

\subsection{Refinement of Hinged Figures}

In this section, we define the important concept of refinement. In its specialization to realizable hinged figures $A$ and $B$, the statement " $A$ is a refinement of $B$ " will imply that $B$ may be obtained from $A$ by gluing part of $A$ 's boundary together and that $A$ may be obtained from $B$ by cutting some of $B$ 's pieces and modifying hinges appropriately. In the further specialization that $B$ is a single polygon, this intuitively means that $A$ is a refinement of $B$ if $A$ has a configuration in the shape of $B$, ignoring all cuts and hinges. We will use this observation to formally define the notion of hinged dissection of polygons.

However, the concept of refinement we define is more general, applying to arbitrary - and not just realizable - hinged figures. The usefulness of our generalized definition of refinement is that it is versatile enough to allow not only regular hinged dissections of polygons, but also a kind of "hinged dissection of hinged figures"; Lemmas 2 and 3 below illustrate this idea.

We call a function $f: \mathcal{T}(A) \rightarrow \mathcal{T}(B)$ a refining map, written $f: A \prec B$ or simply $A \prec B$, and say that $A$ is a refinement of $B$ via $f$, if $f$ satisfies the properties below. (Recall that such a map induces both a mapping of each piece $P$ in $A$ to a region $f(P)$ in $\mathcal{T}(B)$ (not necessarily a whole piece of $B$ ) and induces a mapping of each hinge $h$ in $A$ to a point $f(h)$ in $\mathcal{T}(B)$ (not necessarily a hinge of $B$ ).)

0. $f$ is surjective: $f(\mathcal{T}(A))=\mathcal{T}(B)$.

1. $f$ is isometric and orientation preserving on each piece of $A$ : it maps each piece of $A$ to a congruent subset of one of $B$ 's pieces. Further, every piece of $A$ and its image under $f$ in $\mathcal{T}(B)$ have the same orientation as determined by the (piece) orientations of $A$ and $B$, respectively.

2. The interiors of pieces in $A$ do not overlap in their image under $f$.

3. Cyclic hinge order is preserved. Specifically, consider a hinge $h$ in $A$ incident to pieces $P_{1}, P_{2}, \ldots, P_{p}$. If $f(h)$ is incident to pieces $Q_{1}, Q_{2}, \ldots, Q_{q}$ in $B$ (listed in the order specified by $f(h)$ if $f(h)$ is a hinge of $B$-otherwise, there is just one piece $Q_{1}$ and $m=1$ ), then only a cyclically consecutive interval of pieces $P_{i}, P_{i+1}, \ldots, P_{j}$ (where indices are treated modulo $p$ ) map under $f$ into each piece $Q_{k}$, they appear in counterclockwise order around $f(h)$ in $Q_{k}$, and the concatenation of these intervals in order for $k=1,2, \ldots, q$ consists of all pieces $P_{1}, P_{2}, \ldots, P_{p}$ in that cyclic order.

4. No two hinges of A topologically cross in $B$. For two hinges $h$ and $h^{\prime}$ with $f(h)=$ $f\left(h^{\prime}\right)$, two pieces $P$ and $Q$ incident to $h$ are topologically crossed in $B$ with two pieces $P^{\prime}$ and $Q^{\prime}$ incident to $h^{\prime}$ if the induced cyclic order around $f(h)=f\left(h^{\prime}\right)$ on the four pieces is $f(P), f\left(P^{\prime}\right), f(Q), f\left(Q^{\prime}\right)$ or $f(P), f\left(Q^{\prime}\right), f(Q), f\left(P^{\prime}\right)$. 
Next we illustrate the two key properties of refinements. First, in the special case that $B$ is a realizable hinged figure, a refinement $A \prec B$ corresponds to the intuitive notion that hinged figure $A$ can take on all configurations of $B$. Second, the property of refinement is transitive, even though it is not clear that the concept of "hinged dissection" is itself transitive.

Lemma 2 If $f: A \prec B$ is a refining map, and if $g: B \rightarrow \mathbb{R}^{2}$ is a configuration of $B$, then the map $g \circ f: A \rightarrow \mathbb{R}^{2}$ is a configuration of $A$, called the configuration induced by refinement $f$.

Lemma 3 The property of refinement is transitive: if $f: A \prec B$ and $g: B \prec C$, then $g \circ f: A \prec C$.

Proof of Lemmas 2 and 3 The condition for a map $h_{1}: \mathcal{T}(B) \rightarrow \mathcal{T}(C)$ to be a refinement of $B$ and a map $h_{2}: \mathcal{T}(B) \rightarrow \mathbb{R}^{2}$ to be a configuration of $B$ are nearly identical; the only difference is that $h_{2}$ is mapping into a less complicated topological space and $h_{2}$ is not necessarily surjective. As a result, the proofs of Lemmas 2 and 3 are nearly identical, so we focus on the proof for Lemma 3.

Suppose $f: A \prec B$ and $f: B \prec C$ are refinements. Properties 0-3 of refinement are clearly preserved under composition, so we now verify that $g \circ f$ also has Property 4. Suppose that we have pieces $P$ and $Q$ incident to hinge $h$ and pieces $P^{\prime}$ and $Q^{\prime}$ incident to hinge $h^{\prime}$ as in Property 4. Further suppose for contradiction that the induced cyclic order of these four polygons under $g \circ f$ is $g(f(P)), g\left(f\left(P^{\prime}\right)\right), g(f(Q)), g\left(f\left(Q^{\prime}\right)\right)$; the other ordering is symmetric.

Suppose first that $P$ and $Q$ are mapped via $g \circ f$ into the same piece $R$ of $C$. By the ordering above, $P^{\prime}$ also maps into $R$ between $g(f(P))$ and $g(f(Q))$, while $g\left(f\left(Q^{\prime}\right)\right)$ is either not in $R$ or is not between $g(f(P))$ and $g(f(Q))$ in $R$. Either way, the induced cyclic order of the polygons $f(P), f(Q), f\left(P^{\prime}\right), f\left(Q^{\prime}\right)$ is $f(P), f\left(P^{\prime}\right), f(Q), f\left(Q^{\prime}\right)$, contradicting that $f$ has Property 4.

The same contradiction is reached if $g\left(f\left(P^{\prime}\right)\right)$ and $g\left(f\left(Q^{\prime}\right)\right)$ are in the same piece of $C$, so now suppose that the four polygons $P, Q, P^{\prime}, Q^{\prime}$ map via $g \circ f$ to four distinct pieces $R, S, R^{\prime}, S^{\prime}$ respectively in $C$. Then they also map into distinct pieces $U, V, U^{\prime}, V^{\prime}$ respectively in $B$. If $f(h)$ and $f\left(h^{\prime}\right)$ are in fact the same hinge of $B$, then because $f$ has Property 4, the cyclic order around $f(h)=f\left(h^{\prime}\right)$ is $U, V, U^{\prime}, V^{\prime}$ (without loss of generality, by swapping indices if necessary), and because $g$ has Property 3, the cyclic order around $g(f(h))$ is then $R, S, R^{\prime}, S^{\prime}$, contradicting the assumption that these hinges were crossed in $C$. In the other case, hinges $f(h)$ and $f\left(h^{\prime}\right)$ are separate in $B$, and it follows from Property 4 of $g$ that these hinges map to uncrossed hinges in $C$, which is again contrary to assumption.

If $A$ and $B$ are both realizable hinged figures, then it is clear that $A \prec B$ corresponds to the natural idea that $B$ may be obtained from $A$ by gluing part of $\partial A$ together. In the special case that $B$ is a single polygon, we call a hinged figure $A$ with $A \prec B$ a hinged dissection of $B$. Lemma 2 together with Property 0 show that $A$ can indeed take on the shape of polygon $B$. More generally, a hinged dissection of polygons $P_{1}, P_{2}, \ldots, P_{n}$ is a hinged figure $A$ that is simultaneously a refinement of each $P_{i}, 1 \leq i \leq n$. In particular, hinged dissections are necessarily realizable. 


\section{Universal Hinged Dissection}

In this section, we show (in Theorem 6) that any finite collection of hinged figures $F_{1}, F_{2}, \ldots, F_{n}$ using the same set of pieces has a common refinement $C$ with $C \prec F_{i}$ for all $1 \leq i \leq n$. As a consequence, we obtain (in Corollary 7 ) that any finite collection of equal-area polygons $P_{1}, P_{2}, \ldots, P_{n}$ has a hinged dissection, that is, a hinged figure $C$ that is simultaneously a refinement of (and hence has a configuration in the shape of) each $P_{i}$. At this stage, we do not claim that the common refinement $C$ may be continuously deformed between these configurations; the problem of continuous motion between configurations is addressed later, in Sect. 4.

Theorem 6 follows from two lemmata. First, Lemma 4 describes how a certain type of manipulation called "rooted subtree movement" allows arbitrary reorganization of the pieces of a hinged figure. Second, Lemma 5 shows how to effectively simulate a rooted subtree movement that transforms hinged figure $F$ into $F^{\prime}$ by finding a hinged figure refining both $F$ and $F^{\prime}$, which means by Lemma 2 that the hinged figure can take on all configurations that $F$ and $F^{\prime}$ can. In the end, this simulation will let us prove Theorem 6 and thus Corollary 7.

Mimicking this structure, Sect. 3 is organized as follows. In Sect. 3.1, we define the concept of rooted subtree movement and prove Lemma 4. In Sect. 3.2, we state Lemma 5 and show how the two lemmata may be used to prove Theorem 6 and Corollary 7. Because the proof of Lemma 5 is especially technical, we defer its proof until Sect. 3.4, first giving additional motivating exposition in Sect. 3.3.

\subsection{Rooted Subtree Movement}

Consider a tree-like hinged figure $F$. If there are two disjoint hinged figures $R$ and $S$ with distinguished boundary points $r \in \partial R$ and $s \in \partial S$ such that $F$ is equivalent to the hinged figure obtained by identifying points $r$ and $s$ to a single hinge, we write $F=(R, r) \vee(S, s),{ }^{4}$ and call $(R, r)$ and $(S, s)$ rooted subtrees of $F$. Given a hinged figure $F=(R, r) \vee(S, s)$ and another point $s^{\prime} \in \partial S$, the rooted subtree movement of rooted subtree $(R, r)$ from $(S, s)$ to $\left(S, s^{\prime}\right)$ is an operation transforming $F$ into $F^{\prime}=(R, r) \vee\left(S, s^{\prime}\right)$. Figure 4 shows two examples of rooted subtree movements: transforming (a) into (b), and transforming (b) into (c).

Rooted subtree movements suffice to arbitrarily re-arrange the pieces of any treelike hinged figure:

Lemma 4 For any two tree-like hinged figures $A$ and $B$ with the same set of $k$ pieces, there is a sequence of at most $2 k-2$ rooted subtree movements that transforms $A$ into $B$.

Proof We use induction to prove a slightly more general result: for any two tree-like hinged figures $A$ and $B$, where the $m$ pieces of $A$ form a superset of the $k$ pieces of $B$, there is a sequence of at most $2 k-2$ subtree movements that transforms $A$ into

\footnotetext{
${ }^{4}$ This notation comes from algebraic topology, where this operation of joining two topological spaces at a single point is known as wedge sum $[24$, p. 10] or wedge product [10, p. 136].
} 


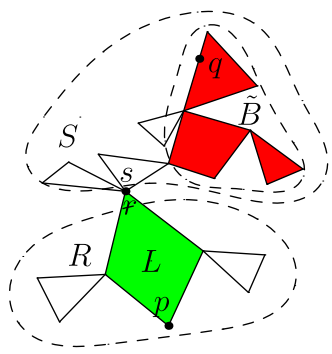

(a) The original hinged figure $(R, r) \vee(S, s)$.

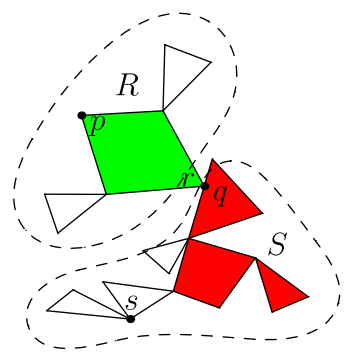

(b) First step: rooted subtree $(R, r)$ has been moved from $(S, s)$ to $(S, q)$.

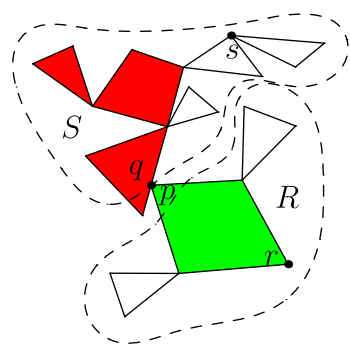

(c) Second step: rooted subtree $(S, q)$ has been moved from $(R, r)$ to $(R, p)$, thus positioning $L$ correctly relative to the other pieces of $B$.

Fig. 4 Rooted subtree movements suffice to arbitrarily re-arrange the pieces of a hinged figure, because they allow one piece to be properly positioned at a time. Here we show how two rooted subtree movements suffice to move the (shaded) piece $L$ into the correct place relative to the other (shaded) pieces $\tilde{B}$ of the target shape $B$

a tree-like hinged figure $A^{\prime}$ containing $B$ as a subfigure. When $m=k$, then $A, A^{\prime}$, and $B$ have the same set of pieces, so $A^{\prime}=B$ and we have transformed $A$ into $B$, proving the lemma.

This stronger result may be proven by induction on $k$. The base case $k=1$ is trivial: performing $2 k-2=0$ subtree movements, we arrive at $A^{\prime}=A$, which certainly contains $B$ (a single polygon) as a subfigure. For $k>1, B$ has a (leaf) piece $L$ attached to only one other piece in $B$. Let $\tilde{B}$ be the subfigure of $B$ formed by the other $k-1$ pieces. By induction, $A$ can be transformed by at most $2(k-1)-2=2 k-4$ subtree movements into a tree-like hinged figure $\tilde{A}$ containing $\tilde{B}$ as a subfigure. It remains to transform $\tilde{A}$ in order to contain $B$ as a subfigure, or in other words, in order to connect $L$ and $\tilde{B}$ so that they form a subfigure identical to $B$.

We use two rooted subtree movements to move $L$ into place. Because $\tilde{A}$ is treelike and $\tilde{B}$ 's pieces are connected in $\tilde{A}$, there is a unique edge in $\tilde{A}$ 's incidence graph incident to $L$ whose removal separates $L$ from $\tilde{B}$ 's pieces. This removal corresponds to a decomposition $\tilde{A}=(R, r) \vee(S, s)$ such that $\tilde{B}$ 's pieces are entirely in $S, L$ is in $R$, and $r$ is a vertex of $L$, as in Fig. 4(a). Let $p$ and $q$ be the boundary points of $L$ and $\tilde{B}$, respectively, that are hinged together in $B$. Now define $A^{\prime \prime}=(R, r) \vee$ $(S, q)$, which may be obtained from $\tilde{A}$ via the rooted subtree movement of $(R, r)$ from $(S, s)$ to $(S, q)$, as in Fig. 4(b). Finally define $A^{\prime}=(R, p) \vee(S, q)$, which may be obtained from $A^{\prime \prime}$ by the rooted subtree movement of $(S, q)$ from $(R, r)$ to $(R, p)$, as in Fig. 4(c).

The two rooted subtree movements transforming $\tilde{A}$ to $A^{\prime}$ fix the pieces of $\tilde{B}$, so these pieces still form a copy of $\tilde{B}$ within $A^{\prime}$. Furthermore, this copy of $\tilde{B}$ is hinged at point $q$ to point $p$ of piece $L$ in $A^{\prime}$, so restricting $A^{\prime}$ to the pieces of $B$ forms a copy of $B$, completing the proof.

Note that Lemma 4 is purely combinatorial in nature; the intermediate hinged figure $\tilde{A}$ may not be realizable, even if $A$ and $B$ are. It is here that we require the 
general combinatorial definition of hinged figures. This nonrealizability will not affect our overall argument, because when we use this lemma we will only need that the final hinged figure $B$ is realizable.

\subsection{Common Hinged Dissections Exist}

The key lemma of our argument is a way to simulate a rooted subtree movement in a hinged figure by refining that figure, in the following precise sense:

Lemma 5 Consider the rooted subtree movement of $(A, a)$ from $(B, b)$ to $\left(B, b^{\prime}\right)$, which transforms hinged figure $F=(A, a) \vee(B, b)$ into $F^{\prime}=(A, a) \vee\left(B, b^{\prime}\right)$, and suppose that $G \prec F$. Then there exists a hinged figure $H$ such that $H \prec G \prec F$ and $H \prec F^{\prime}$.

Intuitively, the use of $G \prec F$ instead of just $F$ itself enables this lemma to be applied repeatedly while only refining. Because of the length and technical detail of the proof of Lemma 5, we give further motivation in Sect. 3.3 and postpone the argument until Sect. 3.4. Subject to Lemma 5, however, we now state and prove the main theorem of Sect. 3:

Theorem 6 For any finite collection of hinged figures $F_{1}, F_{2}, \ldots, F_{n}$ using the same set of pieces, there exists a hinged figure $C$ with $C \prec F_{i}$ for all $1 \leq i \leq n$.

Proof that Lemma 5 implies Theorem 6 The proof proceeds by induction on $n$. The base case $n=1$ is trivial: let $C=F_{1}$. For $n>1$, we can assume by induction that there is a common refinement $C^{\prime}$ of the hinged figures $F_{1}, F_{2}, \ldots, F_{n-1}$. We will show how to further refine $C^{\prime}$ into a hinged figure $C$ so that $C \prec F_{n}$ as well. By Lemma 3, these properties imply that $C \prec F_{i}$ for all $1 \leq i \leq n$.

We construct a common refinement $C$ of $C^{\prime}$ and $F_{n}$ by a combination of Lemmas 4 and 5. Because $F_{1}$ and $F_{n}$ use the same set of pieces, we can apply Lemma 4 to obtain a sequence of rooted subtree movements $M_{1}, M_{2}, \ldots, M_{m}$ that transform $F_{1}$ into $F_{n}$. (In place of $F_{1}$, we could as easily use any $F_{i}$ with $1 \leq i<n$.) Suppose that movement $M_{j}$ transforms hinged figure $K_{j}$ into $K_{j+1}$, where $K_{1}=F_{1}$ and $K_{m+1}=$ $F_{n}$, and let $G_{1}=C^{\prime} \prec F_{1}=K_{1}$. For each movement $M_{j}$ in order, we apply Lemma 5 with $G_{j} \prec K_{j}$ to obtain a hinged figure $G_{j+1}$ such that $G_{j+1} \prec G_{j}$ and $G_{j+1} \prec$ $K_{j+1}$. By the latter property, we can iterate this construction $m$ times, resulting in a hinged figure $G_{m+1}$ such that $G_{m+1} \prec K_{m+1}=F_{n}$ and $G_{m+1} \prec G_{m} \prec G_{m-1} \prec$ $\ldots \prec G_{2} \prec G_{1}=C^{\prime}$ (by Lemma 3). Therefore $C=G_{m+1}$ is the desired common refinement of $F_{n}$ and $C^{\prime}$.

Corollary 7 Any finite collection of polygons $P_{1}, P_{2}, \ldots, P_{n}$ of equal area have a hinged dissection.

Proof By the Lowry-Wallace-Bolyai-Gerwien Theorem [5, 19, 21, 31, 40, p. 222], there exists a common decomposition of $P_{1}, P_{2}, \ldots, P_{n}$ into finitely many polygons $L_{1}, L_{2}, \ldots, L_{k}$. 

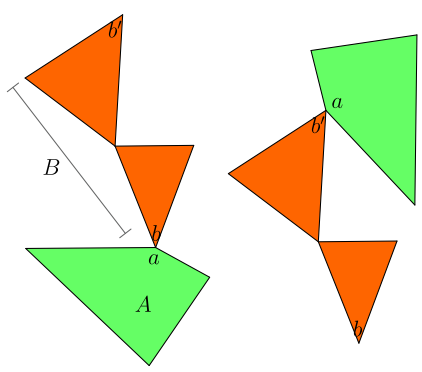

(a) The original hinged figure $F$ (left) and the target $F^{\prime}$ (right).

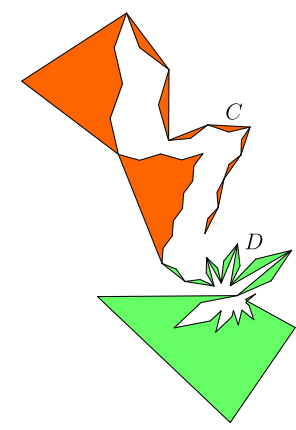

(b) The hinged figure $H$ that refines both $F$ and $F^{\prime}$.
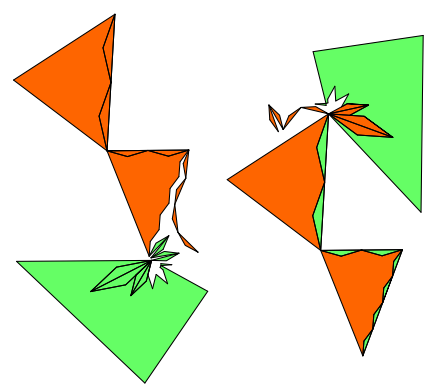

(c) Illustrating the refining maps $H \prec F$ (left) and $H \prec F^{\prime}$ (right).

Fig. 5 The construction for moving a rooted subtree with refinement. Using chains as shown, we may find a common refinement $G$ of two hinged figures $F$ and $F^{\prime}$ related by a rooted subtree movement of $(A, a)$ from $(B, b)$ to $\left(B, b^{\prime}\right)$

For each $i$ with $1 \leq i \leq n$, we construct a tree-like hinging $F_{i}$ of pieces $L_{1}, L_{2}, \ldots, L_{k}$ that refines $P_{i}$ as follows. First we build a spanning tree of the dual graph of the pieces as they arrange to form $P_{i}$. Then, for each edge of this spanning tree, we hinge together the two corresponding pieces at any one shared vertex.

By Theorem 6, we can find a common refinement $C \prec F_{i}$ for all $1 \leq i \leq n$. Combining Lemma 3 together with the fact that $F_{i} \prec P_{i}$, we obtain that $C \prec P_{i}$ for all $1 \leq i \leq n$. Therefore $C$ is the desired hinged dissection.

\subsection{Moving Rooted Subtrees: Motivation}

Here we motivate the construction used to prove Lemma 5. This section is not formally necessary for the proof, but helps clarify the underlying intuition.

Lemma 4 shows that any re-arrangement of a hinged figure's pieces may be obtained via rooted subtree movements. We cannot directly use this result to find common hinged dissections, as hinged dissections and rooted subtree movement are apparently in conflict: hinged dissections do not allow the disconnection of hinges while rooted subtree movements may disconnect hinges.

The goal of Lemma 5 is to resolve this conflict, roughly implementing rooted subtree movement using hinged figures. The key idea in this construction is to connect the two rooted subtrees by a long thin chain of isosceles triangles that "covers" the distance between the broken hinge and the new hinge. In order to create room to "store" this chain, a second chain must be cut away from the boundary path.

Figure 5 illustrates the idea. Consider the hinged figure $F=(A, a) \vee(B, b)$ as in the left of Fig. 5(a), where $A$ is the quadrilateral and $B$ is the union of the two triangles. Suppose we wish to perform the rooted subtree movement of $(A, a)$ from $(B, b)$ to $\left(B, b^{\prime}\right)$, in order to create the hinged figure $F^{\prime}=(A, a) \vee\left(B, b^{\prime}\right)$ on the right of Fig. 5(a). We construct the hinged figure $H$ shown in Fig. 5(b), which constructs the isosceles triangle chains just described. Notice that just removing kites from piece $A$ would result in a polygon that is self-touching and therefore not simple, but this may be fixed by cutting and rehinging a small triangle as shown in Fig. 5(b). The left 
and right sides of Fig. 5(c) respectively illustrate how to give a refining map from $H$ to each of $F$ and $F^{\prime}$, thus establishing that $H$ is a common refinement of $F$ and $F^{\prime}$.

Unfortunately, Lemma 5 is more complicated than what the construction just presented can handle. The issue is that we do not wish to find a common refinement of $F$ and $F^{\prime}$, but rather a common refinement of some refinement $G \prec F$ and $F^{\prime}$. The reason for this is illustrated by our proof of Theorem 6: in order to simulate several consecutive rooted subtree movements on the same set of pieces, we need $G$ to preserve previous refinements.

The full Lemma 5, which moves subtree $(A, a)$ not in $F$ but in its refinement $G \prec F$, has two difficulties. First, the hinge between $a$ and $b$ in $F$ may correspond to multiple hinges in $G$, so we must "break" all of these hinges simultaneously with separate chains. Second, the boundary path between $b$ and $b^{\prime}$ in $F$ may correspond to multiple, disjoint boundary paths in $G$, so we must cut the equivalent of chain $C$ of Fig. 5(b) from this broken boundary path. The details of the full construction, which are along similar lines but distinct from Fig. 5, are formalized in the next subsection.

\subsection{Proof of Lemma 5}

In this section, we give the construction and proof of Lemma 5, completing the proof of Theorem 6 .

\subsubsection{Free Regions}

Our first, rather mundane task is to reserve space along the edges and near the vertices of the pieces in the given hinged figure, for carving out small isosceles triangles to perform rooted subtree movements. We refer to this reserved space as free regions. The free regions consist of small triangles attached to each edge and small circular sectors attached to each vertex. To define these regions, we define the following notation. For an angle $\alpha<90^{\circ}$ and a length $\ell$, let $\triangle_{\alpha}(\ell)$ denote an isosceles triangle with base of length $\ell$ and base angles of $\alpha$. For an angle $\beta$, point $P$, and radius $r$, let $\nabla_{\beta}(P, r)$ be a circular sector (pie wedge) centered at $P$ with angle $\beta$ and radius $r$.

Lemma 8 For any simple, counterclockwise-oriented polygon $V=V_{1} V_{2} \cdots V_{n}$, there exist an angle $\beta$ and a radius $r$ small enough so that the isosceles triangle $\triangle_{\beta}\left(\left|V_{i} V_{i+1}\right|\right)$ inside $V$ with base along the edge $V_{i} V_{i+1}$, and some circular sector $\triangleright_{\beta}\left(V_{i}, r\right)$ drawn inside $V$, for all $i \in\{1,2,3\}$, are pairwise disjoint except at the vertices of $V$.

Throughout the rest of this section, the triangles and sectors defined in Lemma 8 for some suitable $\beta$ and $r$ are called the free regions for their respective edges and vertices of the polygon $V$; see Fig. 6.

Proof We first prove the result for triangles. For a counterclockwise-oriented triangle $T=A B C$ with side lengths $a, b, c$, semiperimeter $s=\frac{1}{2}(a+b+c)$, and angles of $\delta, \epsilon, \zeta$, we choose $\beta_{T}<\frac{1}{3} \min \{\delta, \epsilon, \zeta\}$ and $r_{T}<\min \{s-a, s-b$, $s-c$. We claim that the three triangles $\triangle_{\beta_{T}}(A B), \triangle_{\beta_{T}}(B C)$, and $\triangle_{\beta_{T}}(C A)$ 
Fig. 6 The free regions (lightly shaded) in triangle $A B C$ are separated by the dark circles and the angle trisectors $\ell_{a}, \ell_{a}^{\prime}, \ell_{b}, \ell_{b}^{\prime}, \ell_{c}, \ell_{c}^{\prime}$

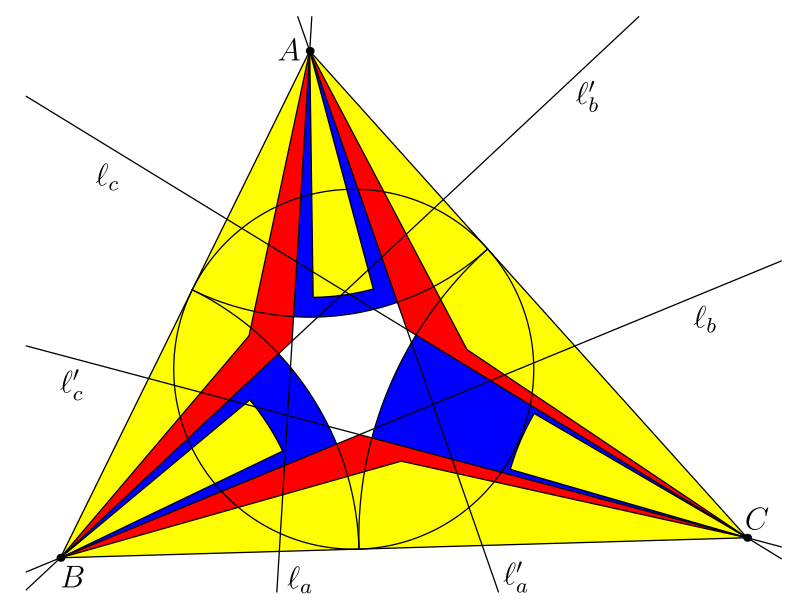

with bases along their respective edges $A B, B C$, and $C A$, along with the three sectors $\triangleright_{\beta_{T}}\left(A, r_{T}\right), \triangleright_{\beta_{T}}\left(B, r_{T}\right), \triangleright_{\beta_{T}}\left(C, r_{T}\right)$, can be drawn in $A B C$ without overlap, as in Fig. 6. Indeed, $\triangle_{\beta_{T}}(A B)$ is contained in the triangle $T_{A}$ between $A B$ and the two trisectors $\ell_{a}$ and $\ell_{b}^{\prime}$, sector $\triangleright_{\beta_{T}}\left(A, r_{T}\right)$ is contained in the sector $S_{A}=\searrow_{\delta / 3}(A, s-a)$ between trisectors $\ell_{a}$ and $\ell_{a}^{\prime}$, and likewise for $B$ and $C$. The six regions $T_{A}, T_{B}, T_{C}, S_{A}, S_{B}, S_{C}$ are interior-disjoint because they are separated by the trisecting lines and darkly shaded circular sectors $\triangleright_{\delta}(A, s-a), \triangleright_{\epsilon}(B, s-b)$, and $D_{\zeta}(C, s-c)$ in Fig. 6. Therefore the claim follows for the six described regions in the triangle $A B C$.

For the case of a general polygon $V=V_{1} V_{2} \cdots V_{n}$, we first triangulate $V=$ $V_{1} V_{2} \cdots V_{n}$ by $n-2$ diagonals. For each triangle $T=V_{i} V_{j} V_{k}$ in the triangulation, calculate $\beta_{T}$ and $r_{T}$ as above, and draw the associated free regions in $T$. Then, as all the resulting triangles and sectors are interior-disjoint by construction, choosing $\beta=\min _{T}\left\{\beta_{T}\right\}$ and $r=\min _{T}\left\{r_{T}\right\}$ suffices.

\subsubsection{Chains}

Next we define a structure similar to the chains of isosceles triangles in Fig. 5. Our new construction, however, will need to use kites in addition to triangles, so we generalize accordingly.

For a sequence of positive lengths $\ell_{1}, \ell_{2}, \ldots, \ell_{n}$ and two angles $\alpha_{1}>\alpha_{2} \geq 0$, we define the chain $\mathcal{C}_{\alpha_{1}, \alpha_{2}}\left(\ell_{1}, \ell_{2}, \ldots, \ell_{n}\right)$ to be the chain-like hinged figure with $2 n$ pieces $M_{1}, M_{2}, \ldots, M_{2 n}$ formed as follows; refer to Fig. 7. The pieces are all similar kites: for $1 \leq i \leq 2 n$, piece $M_{i}=X_{i} W_{i} Y_{i} Z_{i}$ has interior angles of $\alpha_{1}-\alpha_{2}$ at $X_{i}$ and at $Y_{i}, 180^{\circ}+2 \alpha_{2}$ at $W_{i}$, and $180^{\circ}-2 \alpha_{1}$ at $Z_{i}$. The kites differ in their scale, but they come in odd-even pairs of equal size: for $1 \leq i \leq n, M_{2 i-1}$ and $M_{2 i}$ are scaled so that $\left|X_{2 i-1} Y_{2 i-1}\right|=\left|X_{2 i} Y_{2 i}\right|=\ell_{i}$. If $\alpha_{2}>0$, this kite is concave; and if $\alpha_{2}=0$, the kite degenerates into a triangle. For each $1 \leq i<2 n$, kite $M_{i}$ is hinged at vertex $Y_{i}$ to vertex $X_{i+1}$ of the next kite $M_{i+1}$. The initial point $C_{0}$ of the chain $C$ is the vertex $X_{1}$, and the final point $C_{1}$ is the vertex $Y_{2 n}$. When $\alpha_{2}=0$, the chain $\mathcal{C}_{\alpha_{1}, \alpha_{2}}\left(\ell_{1}, \ell_{2}, \ldots, \ell_{n}\right)=\mathcal{C}_{\alpha_{1}, 0}\left(\ell_{1}, \ell_{2}, \ldots, \ell_{n}\right)$ is actually a chain of triangles, so we 


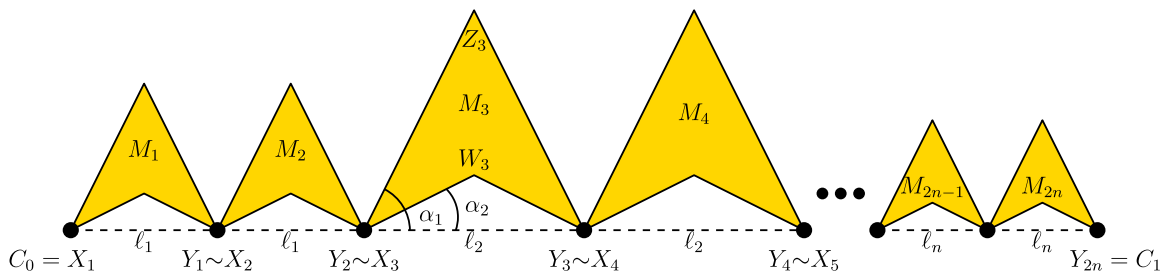

Fig. 7 Definition of the chain $\mathcal{C}_{\alpha_{1}, \alpha_{2}}\left(\ell_{1}, \ell_{2}, \ldots, \ell_{n}\right)$

refer to such a chain as a triangle chain for clarity; likewise, we refer to the chain $\mathcal{C}_{\alpha_{1}, \alpha_{2}}\left(\ell_{1}, \ell_{2}, \ldots, \ell_{n}\right)$ as a kite chain when $\alpha_{2}>0$.

As defined, the chain $\mathcal{C}_{\alpha_{1}, \alpha_{2}}\left(\ell_{1}, \ell_{2}, \ldots, \ell_{n}\right)$ is an abstract hinged figure that admits many configurations in the plane. In the case $\alpha_{2}=0$, one configuration of particular interest places $C_{0}=X_{1}, Y_{2} \sim X_{3}, Y_{4} \sim X_{5}, \ldots, Y_{2 n-2} \sim X_{2 n-1}, Y_{2 n}=C_{1}$ at a common point, and packs the odd-even kite pairs tightly around this point in cyclic order, so that $M_{2}$ shares an edge with $M_{3}, M_{4}$ shares an edge with $M_{5}$, and so on. We call this configuration a triangle sweep and denote it by $\mathcal{C}_{\alpha_{1}, 0}^{+}\left(\ell_{1}, \ell_{2}, \ldots, \ell_{n}\right)$. This configuration looks like a collection of $n$ abutting rhombi organized cyclically around a point, as in Figs. 5 and 9.

\subsubsection{Construction}

We may now finally prove Lemma 5, which we restate here for reference.

Lemma 5 Consider the rooted subtree movement of $(A, a)$ from $(B, b)$ to $\left(B, b^{\prime}\right)$, which transforms hinged figure $F=(A, a) \vee(B, b)$ into $F^{\prime}=(A, a) \vee\left(B, b^{\prime}\right)$, and suppose that $G \prec F$. Then there exists a hinged figure $H$ such that $H \prec G \prec F$ and $H \prec F^{\prime}$.

The construction proceeds in three steps. First, we identify the pieces adjacent to the points $a$ and $b$. Then, we cut a chain along the boundary of $B$. Finally, we cut a chain from $A$, which may be interchanged with the boundary chain as illustrated in Figs. 8, 9.

Proof First note that, because $A$ and $B$ are subfigures of a tree-like figure $F, A$ and $B$ are themselves tree-like. Thus, the boundary $\partial B$ is connected, and there are exactly two boundary paths from $b$ to $b^{\prime}$. Choose the path $\gamma$ that winds counterclockwise around the boundary of $B$. Also choose a refining map $f: G \prec F$.

Step 1: Locate the pieces connecting $(A, a)$ and $(B, b)$. Consider the behavior of $G$ near the hinge $h$ of $F$ connecting points $a$ and $b$ : let $h_{1}, h_{2}, \ldots, h_{n}$ be all hinges of $G$ with the property that $h_{i}$ has two incident pieces that map under $f$ into $A$ and $B$ respectively. (In other words, hinges $h_{1}, h_{2}, \ldots, h_{n}$ are the refinement in $G$ of hinge $h$ in $F$.)

Because pieces are by definition simple polygons, any piece of $G$ is incident to at most one of $h_{1}, \ldots, h_{n}$. For each $i$, let $L_{i}^{a}$ and $L_{i}^{b}$ be two pieces incident to $h_{i}$ so 


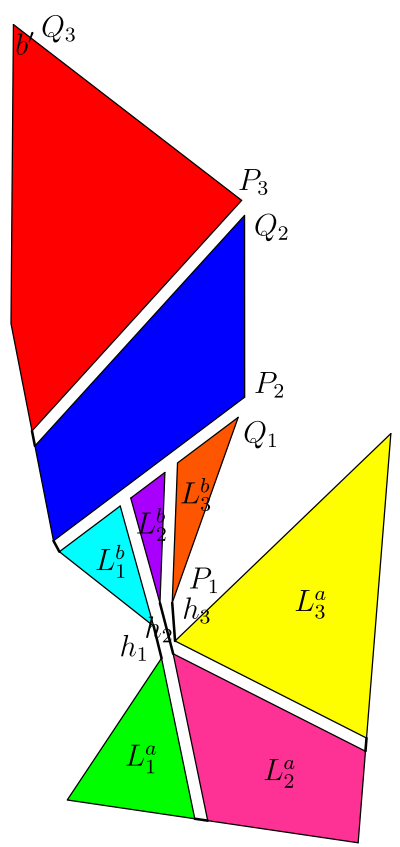

(a) The hinged figure $G$ arranged to illustrate its refining $\operatorname{map} G \prec$ $F$ for a two-pieced hinged figure $F$.

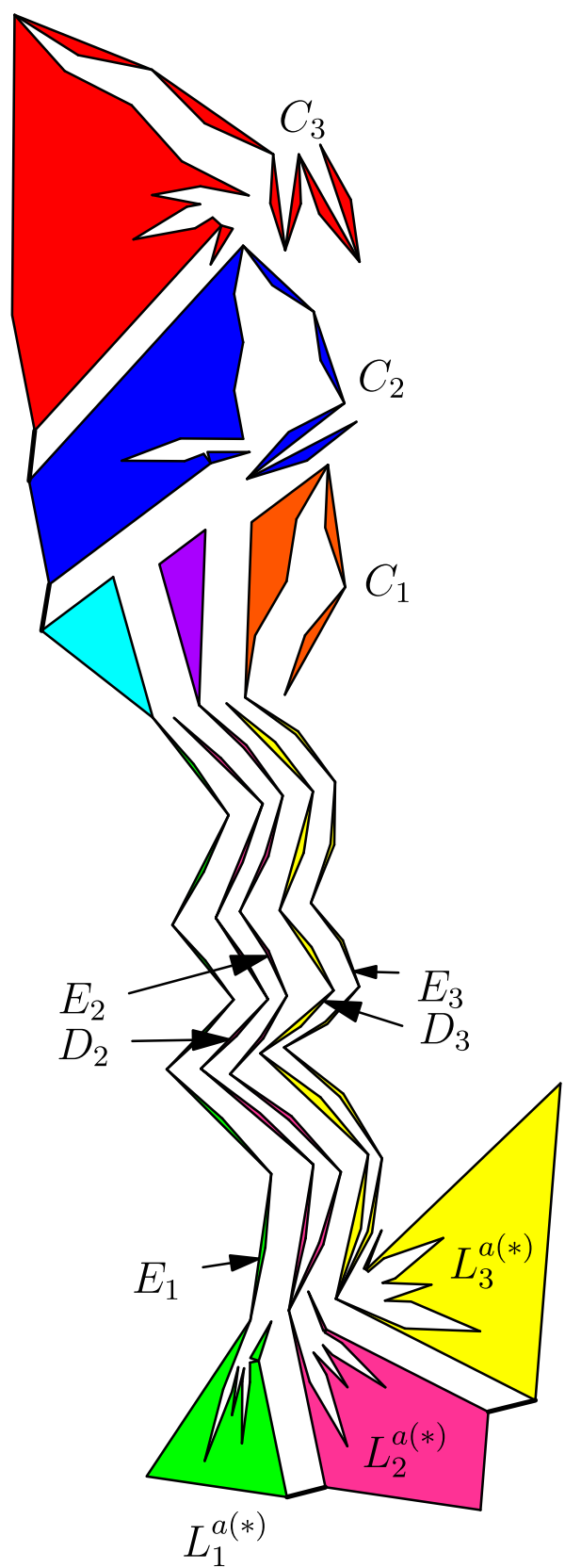

(b) The constructed common refinement of $G$ and $F^{\prime}$. Step 2 constructs the triangle chains $C_{i}$, and Step 3 constructs the triangle chains $D_{i}$ and kite chains $E_{i}$.

Fig. 8 The construction for Lemma 5, illustrating its refinement of $G$ 
Fig. 9 The construction for Lemma 5, illustrating its refinement of $F^{\prime}$

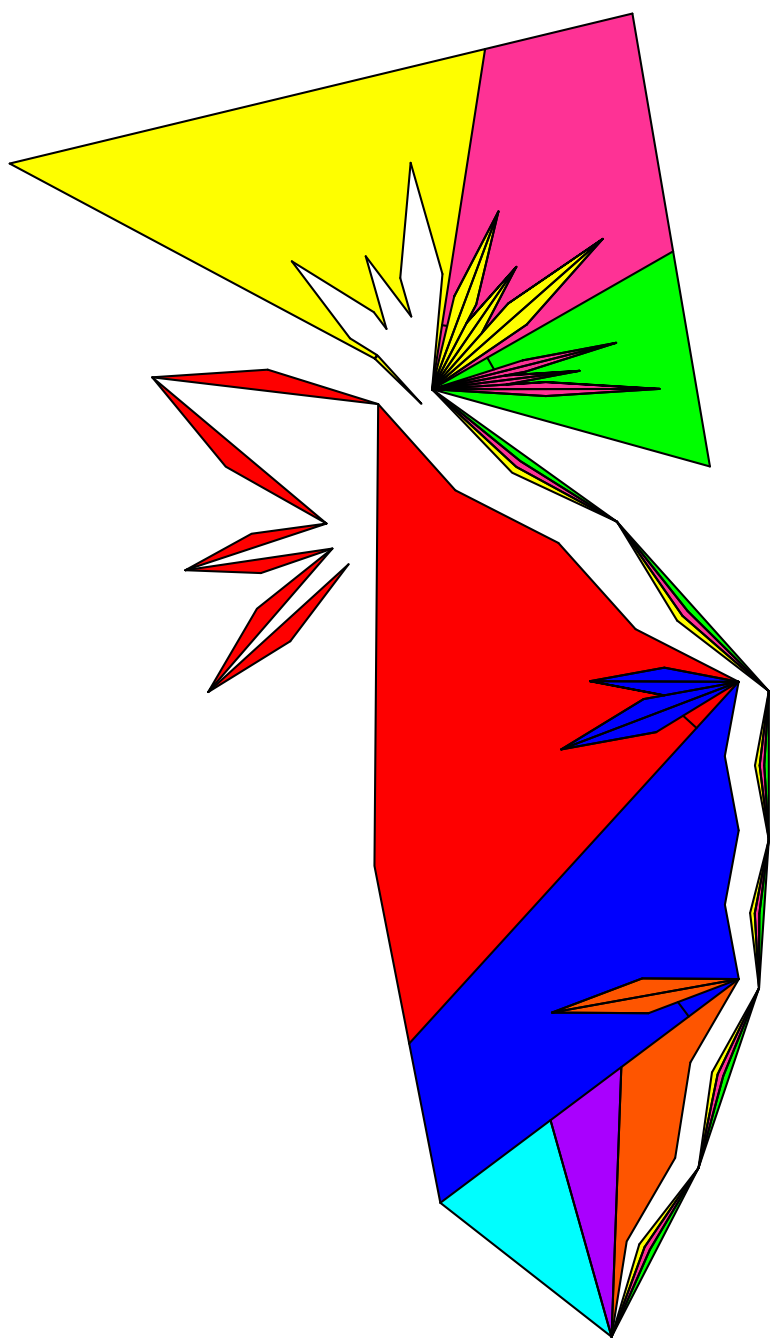

that $f\left(L_{i}^{a}\right) \subset A$ and $f\left(L_{i}^{b}\right) \subset B$; if there are more than two pieces incident to $h_{i}$, we may choose any pieces $L_{i}^{a}$ and $L_{i}^{b}$ with the above description, and the extra pieces will not affect the construction below. Without loss of generality, we may assume that these pieces have been numbered so that their induced cyclic counterclockwise order around $h$ is $f\left(L_{1}^{a}\right), f\left(L_{2}^{a}\right), \ldots, f\left(L_{n-1}^{a}\right), f\left(L_{n}^{a}\right), f\left(L_{n}^{b}\right), f\left(L_{n-1}^{b}\right), \ldots, f\left(L_{1}^{b}\right)$.

Step 2: Cut a chain along the boundary path. We first add some extra $180^{\circ}$ vertices to the boundary $\partial G$ : Namely, any boundary point $p \in \partial G$ that is collocated (via map $f$ ) with a vertex of any piece in $G$ will itself be declared a (possibly $180^{\circ}$ ) vertex of its piece. We also declare $b^{\prime}$ to be a vertex of its piece if it is not already.

The path $\gamma$ is defined to follow $\partial B$. The set $f^{-1}(\gamma)$ (where $f: G \prec F$ is the refining map from above) is contained in $\partial G$, and is the union of finitely many edges and vertices of $\partial G$. Let $\Gamma$ be the collection of subpaths along $\partial G$ formed by these 
edges of $f^{-1}(\gamma)$. Informally, these paths in $\Gamma$ follow the boundary $\partial G$ for a while, until skipping over a portion of $\partial G$ interior to $B$, then returning to following a portion of $\partial G$, and so on. Let $S_{1}, S_{2}, \ldots, S_{s}$ denote the maximal portions of $\Gamma$ that follow $\partial G$ without skipping, in order, so that in particular $\Gamma$ is the concatenation of these portions, or equivalently, $\gamma$ is the in-order concatenation of $f\left(S_{1}\right), \ldots, f\left(S_{S}\right)$. More precisely, the transitions from $S_{j}$ to $S_{j+1}$ are precisely where the last vertex of $S_{j}$ does not match the first vertex of $S_{j+1}$ because they are separated by a positive distance in $\partial G$.

Next we define some of the parameters for a chain construction along $\Gamma$. Apply Lemma 8 to obtain a free-region radius and angle for each piece in $G$ incident to $\Gamma$. Let $r$ and $\alpha$ be the smallest such radius and angle respectively. We repeatedly subdivide each edge of each $S_{i}$ of length more than $2 r$ at its midpoint, so that all resulting edges have length at most $2 r$. Let $t$ denote the total number of edges along $\Gamma$ after this subdivision. Let $2 \ell(e) \leq 2 r$ denote the length of each edge $e$ along $\Gamma$, and for any subsequence $S$ of edges in $\Gamma$, let $\ell(S)$ denote the sequence of $\ell(e)$ for each edge $e$ along $S$ in order. Also let $\beta=\alpha /(2 t)$.

Now, for each $1 \leq j \leq s$, we separately construct a chain for $S_{j}$. For each edge $e$ along $S_{j}$, we cut two $\triangle_{\beta}(\ell(e))$ triangles inward in the piece with their bases each spanning a disjoint half of $e$. Because $\beta$ is less than the free-region angle $\alpha$ along each edge along the path $S_{j}$, all these removed isosceles triangles fit within their pieces. We rehinge these removed triangles into a triangle chain $\mathcal{C}_{\beta, 0}\left(\ell\left(S_{j}\right)\right)$ and attach it to $G$ by hinging its final point to the last vertex of $S_{j}$ (as it was before).

Next, for each $2 \leq j \leq s$, we cut a triangle sweep $\mathcal{C}_{\beta, 0}^{+}\left(\ell\left(S_{1}\right), \ell\left(S_{2}\right), \ldots, \ell\left(S_{i-1}\right)\right)$ from the free sector at the first vertex of $S_{j}$, and then make the incident piece of $G$ simple by removing and rehinging a small corner as shown in Fig. 8(b). This triangle sweep has total angle $2 \beta\left(\left|S_{1}\right|+\left|S_{2}\right|+\cdots+\left|S_{i-1}\right|\right) \leq 2 t \beta=\alpha$, and the largest triangle has diagonal $\max \ell\left(S_{1}, S_{2}, \ldots, S_{i-1}\right) \leq r$, so the triangle sweep fits in the free sector. We attach the final point of the triangle sweep $\mathcal{C}_{\beta, 0}^{+}\left(\ell\left(S_{1}\right), \ell\left(S_{2}\right), \ldots, \ell\left(S_{i-1}\right)\right)$ to the initial point of the previously constructed triangle chain $\mathcal{C}_{\beta, 0}\left(\ell\left(S_{j}\right)\right)$, which together form the triangle chain $C_{j}$ indicated in Fig. 8(b).

To see that this construction still refines $G$, note that each of the $s$ triangle chains $C_{1}, C_{2}, \ldots, C_{s}$ may simply fill in the spaces from which they were cut.

Step 3: Cut a chain at hinge $h$. Now we show how to refine $G$ around hinges $h_{1}, h_{2}, \ldots, h_{n}$.

For each $1 \leq i \leq n$, we cut a triangle sweep $\mathcal{C}_{i \beta / n, 0}^{+}(\ell(\Gamma))$ in the free region in $L_{i}^{a}$ at $h_{i}$. The resulting nonsimple piece has two corners at $h_{i}$, so we cut off and rehinge the more counterclockwise of the two, denoting the resulting piece (without this small corner) by $L_{i}^{a(*)}$. As before, by our choice of $r$ and $\beta$, the $i$ th kite sweep can fit within the free sector of $L_{i}^{a}$ at $h_{i}$.

Next, for $2 \leq i \leq n$, we cut each of the $2 t$ triangles $\triangle_{i \beta / n}(\ell(e))$ removed from $L_{i}^{a}$ into two pieces: a slightly smaller triangle $\triangle_{(i-1) \beta / n}(\ell(e))$ with the same base, and the remaining kite whose four angles are $\beta / n, 180^{\circ}+2(i-1) \beta / n, \beta / n$, and $180^{\circ}-$ $2 i \beta / n$. We hinge the new triangles into a new triangle chain $D_{i}=\mathcal{C}_{(i-1) \beta / n, 0}(\ell(\Gamma))$, and hinge the kites into a kite chain $E_{i}=\mathcal{C}_{(i-1) \beta / n, i \beta / n}(\ell(\Gamma))$, as in Fig. 8(b). For $i=1$, we hinge the uncut triangles into the triangle chain $\left.E_{1}=\mathcal{C}_{\beta / n, 0}(\ell(S))\right)$, while 
$D_{1}$ does not exist. For $1 \leq i \leq n$, we attach the initial point of $D_{i}$ (if it exists) and the initial point of $E_{i}$ to hinge $h_{i}$ of $L_{i}^{a(*)}$, and we attach the final point of $E_{i}$ to hinge $h_{b}$.

As before, these modifications result in a refinement of $G$ because each piece may take its original position. This completes our construction of the desired refinement $H \prec G$.

Refining $F^{\prime}$. It remains to show that this hinged figure $H$ is also a refinement of $F^{\prime}$. To do so, we describe the intended alternate configuration of $H$ shown in Fig. 9. For $2 \leq i \leq n$, chain $D_{i}$ fills in the triangle sweep of $L_{i-1}^{a}$, while $L_{n}^{a}$ 's triangle sweep remains unfilled. The kite chains $E_{1}, E_{2}, \ldots, E_{n}$ fit together to form a refinement of a triangle chain $\mathcal{C}_{\beta, 0}(\ell(\Gamma))$, which fills in the triangle chains $\mathcal{C}_{\beta, 0}\left(\ell\left(S_{1}\right)\right), \mathcal{C}_{\beta, 0}\left(\ell\left(S_{2}\right)\right)$, $\ldots, \mathcal{C}_{\beta, 0}\left(\ell\left(S_{S}\right)\right)$ taken from edges along $\Gamma$. For $1 \leq i<s$, the chain $C_{i}$ fills in the triangle sweep $\mathcal{C}_{\beta, 0}^{+}\left(\ell\left(S_{1}\right), \ell\left(S_{2}\right), \ldots, \ell\left(S_{i}\right)\right)$ taken from the first vertex of $S_{i+1}$. Finally, chain $C_{s}$ fills in the unfilled triangle sweep of $L_{n}^{a}$. This is exactly the desired form of $F^{\prime}$, so we are done.

\section{Continuous Motion}

Corollary 7 constructs a hinged dissection that has a configuration in the form of each of $n$ polygons. This section shows how to further refine that hinged dissection to enable it to fold continuously into each polygon while avoiding intersection among the pieces. In other words, this section proves that the questions of common hinged dissection in the wobbly hinged and the continuous models are equivalent. This equivalence follows directly from the main theorem of this section:

Theorem 9 Any hinged figure $A$ has a refinement $B \prec A$ so that any two configurations of $B$ are reachable by a continuous non-self-intersecting motion. The number of pieces in $B$ is at most thrice the total number of vertices of pieces in $A$.

Given polygons $P_{1}, P_{2}, \ldots, P_{n}$ of equal area, Corollary 7 guarantees that they have a hinged dissection $F$. By Theorem 9, there is a refinement $F^{\prime} \prec F$ that is universally reconfigurable without self-intersection. In particular, $F^{\prime}$ can continuously deform between any of the configurations induced by $P_{1}, P_{2}, \ldots, P_{n}$. This hinged figure $F^{\prime}$ solves the problem, proving the first sentence of Theorem 1.

To prove Theorem 9, we require two preliminary results. The first deals with polygonal chains and slender adornments (Sect. 4.1), and the second chainifies a given hinged figure (Sect. 4.2).

\subsection{Slender Adornments}

Slender adornments are defined by Connelly et al. [7]. An adornment is a connected, compact region together with a line segment $A B$, called the base, lying nonstrictly inside the region. Furthermore, the two boundary arcs from $A$ to $B$ must be piecewise differentiable, with one-sided derivatives existing everywhere. An adornment is slender if, for every point $P$ on the boundary other than $A$ and $B$, the primary inward normals at $P$-that is, the rays from $p$ perpendicular to the one-sided derivatives 
at $p$-intersect the base segment $A B$ (possibly at the endpoints). For example, a triangle $A B C$ whose angle at the vertex $C$ opposite the base $A B$ is nonacute $\left(\geq 90^{\circ}\right)$ is slender [7].

In [7], it is shown that certain chains of slender adornments are universally reconfigurable. Specifically, a strictly simple polygonal chain has the property that edges intersect each other only at common endpoints.

Theorem 10 [7, Theorem 8] A strictly simple polygonal chain adorned with slender adornments to either side can always be straightened or convexified.

This theorem implies that any strictly simple open chain adorned with slender adornments is universally reconfigurable: to find a continuous motion between two configurations $c_{1}$ and $c_{2}$, we can simply follow a motion from $c_{1}$ to the straight configuration $c$, and then reverse a motion from $c_{2}$ to $c$.

As we will see in Sect. 4.2, it is relatively easy to refine a hinged dissection into a chain of slender adornments. Unfortunately, however, it is more difficult to obtain the property of strict simplicity. Therefore we turn to a recent generalization of Theorem 10 obtained in [1]. Specifically, an adornment is strictly slender if, for every point $P$ on the boundary other than $A$ and $B$, the inward normals at $P$ intersect the relative interior of the base segment $A B$ (excluding the endpoints). For example, a triangle $A B C$ whose angle at the vertex $C$ opposite the base $A B$ is strictly obtuse $\left(>90^{\circ}\right)$ is strictly slender.

Theorem 11 [1, Theorem 4] Any open polygonal chain adorned with strictly slender adornments to either side can always be straightened.

This theorem drops the condition of strict simplicity but adds the requirements that the chain be open and that the adornments be strictly slender. Fortunately, these conditions are much easier for us to attain.

\subsection{Chainification}

Next, we prove that any hinged figure has a refinement that is chain-like and adorned with (strictly slender) strictly obtuse triangles:

Theorem 12 Any hinged figure $F$ has a chain-like refinement $G \prec F$ so that $G$ consists of a chain of similarly oriented, strictly obtuse triangles hinged at their acuteangled vertices. The number of pieces in $G$ is at most thrice the total number of vertices of pieces in $F$.

Proof Refer to Fig. 10. First we refine $F$ to consist of a tree of triangles hinged at vertices, as follows. For each $n$-sided piece $L$ with $n \geq 4$, draw a collection of triangulating diagonals. Sequentially, for each such diagonal $V_{1} V_{i}$ currently in piece $V=V_{1} V_{2} \cdots V_{k}$ (which may be a refinement of an original piece), replace $V$ with two pieces $V_{1} V_{2} \cdots V_{i}$ and $V_{i} V_{i+1} \cdots V_{1}$ hinged at $V_{1}$, attaching the hinge originally at $V_{i}$ to its corresponding position on either refined piece. The resulting figure consists of 
Fig. 10 Chainifying a hinged figure. We first refine each piece into triangles and detach hinges to make the figure tree-like (middle), and then we cut each triangle into three smaller triangles at the incenter to form a chain-like hinged figure (right)

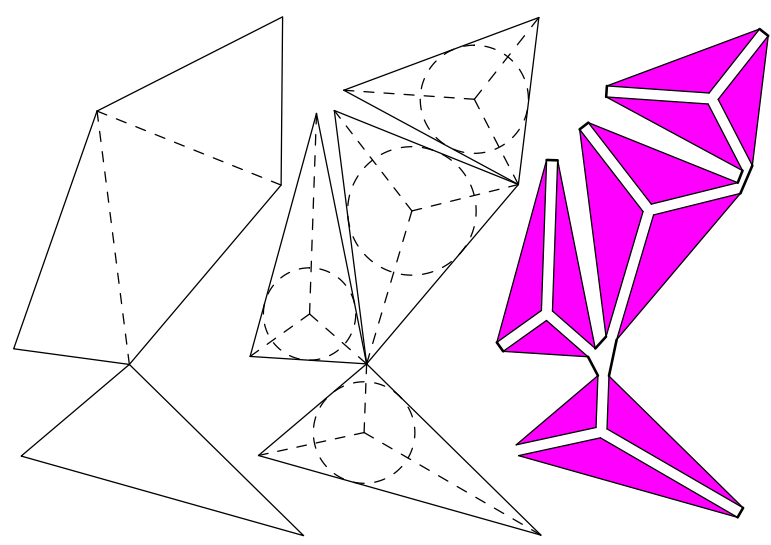

triangles hinged at vertices, with the number of triangles at most the total number of vertices of pieces in $G$.

Next, if the resulting triangulated figure is not tree-like, we repeatedly remove an edge from a cycle in the incidence graph (i.e., remove the corresponding piece from its hinge) until the graph becomes tree-like. Call this refinement $H$.

For each triangular piece $A B C$ in $H$, divide $A B C$ into three triangles $A I B, B I C$, $C I A$, where $I$ is the incenter of $\triangle A B C$. Note that $\angle B I C=180^{\circ}-\frac{1}{2} \angle B-\frac{1}{2} \angle C>$ $180^{\circ}-\frac{1}{2}(\angle A+\angle B+\angle C)=90^{\circ}$, i.e., $\angle B I C$ is strictly obtuse, and likewise for the others. Finally, by hinging these strictly obtuse triangles at the base vertices by walking around $H$ 's boundary, we obtain the desired chain-like refinement $G$.

Now it is easy to prove Theorem 9, i.e., that any hinged figure $A$ has a universally reconfigurable refinement $B$.

Proof of Theorem 9 We apply Theorem 12 to refine the given hinged figure $A$ into a chain-like hinged figure $B$ consisting of strictly obtuse triangles hinged along their bases. Thus $B$ is an open chain adorned with strictly obtuse adornments. Therefore Theorem 11 applies, and we obtain a motion from any configuration of $B$ to a common (straight) configuration, proving universal reconfigurability.

\section{Pseudopolynomial Bounds}

Our algorithm can produce hinged dissections with an exponential number of pieces, as the number of triangle chains required to move a rooted subtree increases with the complexity of the current refinement, as in Figs. 8, 9. Thus, depending on the specific sequence of subtree movements (Lemma 4), sequential refinements can more than double the complexity of the figure at each step.

In the special case that the target polygons can be drawn on a rational grid, however, we can do better. We now describe how to combine the preceding steps with ideas of Eppstein [18] and the classic rectangle-to-rectangle dissection of Montucla 
[35] to perform a hinged dissection using only a pseudopolynomial (that is, polynomial in the dimensions of the grid) number of pieces, proving the second sentence of Theorem 1. In contrast to Theorem 6, this transformation applies only to hinged dissections between exactly two polygons lying on a common grid, rather than to arbitrarily many arbitrary target shapes.

\subsection{Motivation and Overview}

The overall idea is as follows. The general construction is inefficient because movements may traverse the same boundary segments many times, leading to a recursive application of the construction in Lemma 5 and causing exponentially many interconnections as a result of the layered triangle chains. By performing some initial simplifying steps, we instead ensure that the recursion is of constant depth.

At a high level, our algorithm triangulates the polygons into hinged chains of equal-area triangles, as described in Sect. 5.2, and then hinge-dissects between corresponding pairs of triangles while preserving the vertices at which adjacent triangles interconnect. The latter triangle-to-triangle hinged dissection mimics the classic construction for general (unhinged) dissections by Lowry [19, 31], and proceeds in two stages. The first stage is a simple hinged dissection from any triangle to some rectangle, given in Sect. 5.4, which we use on both triangles to reduce to a rectangle-torectangle problem. The second stage is a hinged version of the rectangle-to-rectangle dissection of Montucla [35]; see Sect. 5.5. But for the whole construction to work, we need to compose these hinged dissections sequentially so that our figure refines all the intermediate steps. With unhinged dissections, this composition is easy: just overlay the cuts. The apparent nontransitivity of hinged dissections has always been a stumbling block in this approach.

In Sect. 5.6, we show how to compose hinged dissections while preserving all existing hinges and foldings using a new tool called pseudocuts. Effectively, this tool lets us add a cut and a hinge through an existing hinged figure by only refining that figure (similar to how Lemma 5 allows us to perform subtree movement even if we are actually working with a refinement of the input figure). The tool is built out of iterated rooted subtree movement, modified somewhat for efficiency.

For the preceding constructions to work efficiently, we need to perform our hinged dissections on each pair of triangles as though they existed in isolation, in the sense that dissection operations on one triangle should not affect the rest of the triangle chain. This isolation would fail, for example, if we performed a rooted subtree movement on a single triangle, but the boundary path between the source and destination points crossed outside that triangle and followed the boundary of the rest of the chain. To prevent this, at any point in our construction where a movement or similar operation would follow a boundary path outside the current triangle, we first perform a rooted subtree movement, moving the hinge joint that connects the two adjacent triangles so that it no longer lies on (the interior of) the boundary that will be traversed. The specific movement operations are specified on a case-by-case basis in the sections that follow whenever an operation might manifest this conflict.

However, attempting to preserve isolation in this way causes a further conflict: the intention is to prevent operations on one triangle from altering its neighbors, but 
the subtree movement of Lemma 5 modifies the subtree being moved, which in this case is the neighboring triangle itself. To resolve this and maintain full isolation between distinct triangles, we develop one additional tool, the unaltered rooted subtree movement of Sect. 5.3, which is a modification to rooted subtree movement with the additional property that it does not modify the pieces within the moved rooted subtree. This allows the previously described steps to be performed on each triangle in isolation. Because of the necessity of this tool throughout our construction, we present it rather early in this section.

Unaltered rooted subtree movement also provides a way to obtain the one remaining property needed for our hinged dissection. The steps so far do not guarantee that the attachment vertices of one triangle map to the attachment vertices of the other, which is required for a common refinement of the chains. Using unaltered rooted subtree movement, we can perform the final step of moving the hinge joint that connects two adjacent triangles so that it lies at the correct boundary point of each of those triangles, satisfying this final requirement.

At the very end, we apply Theorem 9 to guarantee continuous motion between the input configurations.

In what follows, we describe the many pieces of this algorithm, followed in Sect. 5.7 by a detailed description of the algorithm and a proof of our pseudopolynomial bound.

\subsection{Triangulation}

For simplicity, we assume by suitable scaling that both polygons are initially drawn on the integer grid. Our first step reduces the input polygons to two chains of equalarea triangles. Specifically, we need two chains with an equal number of triangles, where the $i$ th triangles in both chains have the same area. To achieve this, we will simply guarantee that all triangles in both chains have the same area. We also obtain the property that all vertices in the resulting figure lie on the $\frac{1}{3}$-integer grid, which is useful in our efficiency analysis.

Lemma 13 Every polygon whose vertices lie on the integer grid can be refined into a hinged chain of area- $\frac{1}{6}$ triangles in such a way that every vertex in the chain lies on the $\frac{1}{3}$-integer grid.

Proof First we triangulate the polygon using its vertices and all grid points within its boundaries (inclusive). Every triangle in such a triangulation has three boundary grid points and zero interior grid points, so by Pick's Theorem [23, 37], has area exactly $\frac{1}{2}$. We then chainify these triangulations similar to Theorem 12 , but cutting to the centroid rather than the incenter. Using the centroid means that the resulting triangles may not be obtuse (as they were in Theorem 12), but guarantees that the smaller triangles trisect the area. Because the choice of interior point does not affect the topology of the construction, the result is still a chain. Thus, this process reduces the polygon to a chain of triangles, all with identical area $\frac{1}{6}$. The vertices are integer grid points and centroids of triangles lying on the integer grid, which lie on the $\frac{1}{3}$ integer grid as required. 
Given these chains of equal-area triangles, our task reduces to describing a hinged dissection between each pair of triangles in such a way that the connecting vertices of one triangle map to the connecting vertices of the other triangle. Eppstein [18] originally proposed this approach for proving the existence of hinged dissections. Here we use it to reduce the number of pieces required: if the hinged dissection for each pair of triangles requires only pseudopolynomially many pieces, then the overall hinged dissection requires at most a pseudoquadratic factor more (quadratic in the original grid dimensions), because the area of the input polygons themselves must be at most pseudoquadratic.

\subsection{Unaltered Rooted Subtree Movement}

In Lemma 5, we showed that subtrees in a hinged figure could be moved not just in a hinged figure $F$, but also in any refinement $H \prec F$, allowing effective commutativity in subtree movements. For our pseudopolynomial construction, we find it useful to be able to perform rooted subtree movements without modifying the pieces in one of the subtrees. By contrast, recall that our construction in Lemma 5 cuts a kite sweep out of one subtree and cuts a triangle chain out of the other subtree. Here we describe a modification to Lemma 5 that performs all changes in one subtree.

Lemma 14 Consider the rooted subtree movement of $(A, a)$ from $(B, b)$ to $\left(B, b^{\prime}\right)$, which transforms hinged figure $F=(A, a) \vee(B, b)$ into $F^{\prime}=(A, a) \vee\left(B, b^{\prime}\right)$, and let $G \prec F$ be a refinement of $F$. Then there exists a hinged figure $H$ such that $H \prec$ $G \prec F, H \prec F^{\prime}$, and the subtree $A$ in $H$ is unaltered, that is, the refinement $H \prec G$ is the identity function on the inverse image of $A$.

The proof is identical to a special case of Lemma 5, with some minor modifications. We first describe the intuition for these modifications, followed below by the explicit formal changes.

In the original lemma, we cut a kite sweep out of the subtree $(A, a)$, which extended into a long triangle chain reaching all the way from the source point $b$ to the destination point $b^{\prime}$. At the same time, we cut a series of triangle chains out of the traversed boundary $\gamma$, connecting them to the point furthest along $\gamma$ and making each subsequent chain longer so that, when the subtree $A$ had been moved, the chain at $b^{\prime}$ could fill in the empty space left by $A$ 's kite sweep.

In order to avoid modifying $A$, however, we will instead cut the kite sweep out of (the free region at) $\left(B, b^{\prime}\right)$, connecting it to the base point $a$. Then, when $A$ has reached the destination point, there is an empty kite sweep at the source point $b$ that needs to be filled in (rather than at $a$, as before). We therefore reverse the direction of the boundary kite chains, so that they connect to the earliest point along $\gamma$, with the shortest chain at the end of $\gamma$ and preceding chains growing longer so that the largest chain connects to $b$ and can fill in the empty region left by the kite sweep.

Proof of Lemma 14 We modify the proof of Lemma 5 as follows. First, in Step 2, we re-attach the removed triangle chains by hinging its first (rather than final) vertex to the first (rather than last) vertex of $S_{j}$. Second, also in Step 2, for each $1 \leq j \leq s-1$, 


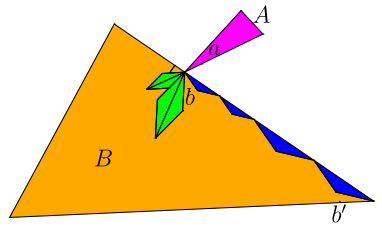

(a)

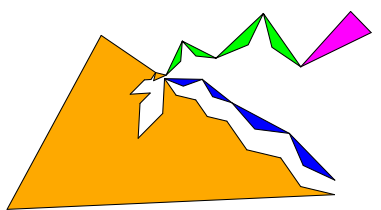

(b)

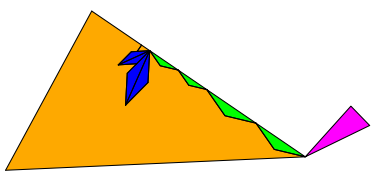

(c)

Fig. 11 Moving the subtree $(A, a)$ from $(B, b)$ to $\left(B, b^{\prime}\right)$ by modifying only the parent tree $B$

Fig. 12 Three-piece hinged dissection of any triangle into some rectangle

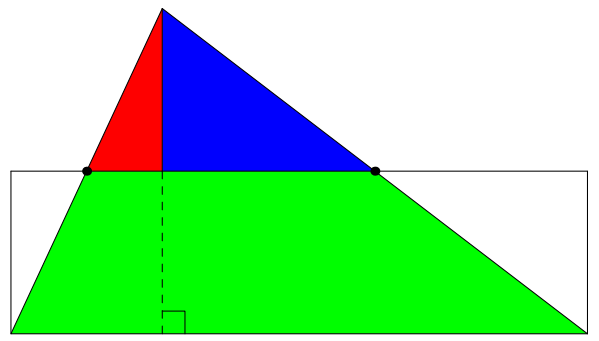

we cut a triangle sweep $\mathcal{C}_{\beta, 0}^{+}\left(\ell\left(S_{s}\right), \ell\left(S_{s-1}\right), \ldots, \ell\left(S_{i+1}\right)\right)$ (rather than for $2 \leq j \leq s$ cutting $\left.\mathcal{C}_{\beta, 0}^{+}\left(\ell\left(S_{1}\right), \ell\left(S_{2}\right), \ldots, \ell\left(S_{i-1}\right)\right)\right)$. Finally, in Step 3, we cut the triangle sweep in the free region in $L_{i}^{b}$ (rather than $L_{i}^{a}$ ), so it does not modify the subtree being moved. With these substitutions, the remainder of the proof proceeds unchanged from that of Lemma 5. Figure 11 shows an example of these modifications.

\subsection{Triangle to Rectangle}

The classic construction for general (unhinged) dissections by Lowry [19, 31] reduces the problem of triangle-to-triangle dissection to that of rectangle-to-rectangle dissection by converting each triangle into a rectangle. In fact, this classical three-piece dissection can be hinged, as we now illustrate.

Lemma 15 There is a three-piece hinged dissection from any triangle to some rectangle.

Proof See Fig. 12. Define the base of a triangle to be the side opposite the obtuse angle, if there is one, or else any side of the triangle. Cut parallel to the base at half the height of the triangle. Cut perpendicular from this line to the apex of the triangle (opposite the base). Hinge the two triangular pieces at the boundary points so that they rotate down to fill a rectangle of half the height of the triangle.

\subsection{Rectangle to Rectangle}

Next we describe a pseudopolynomial hinged dissection between any two rectangles of equal area. Roughly speaking, we start with the classic (unhinged) dissection of 

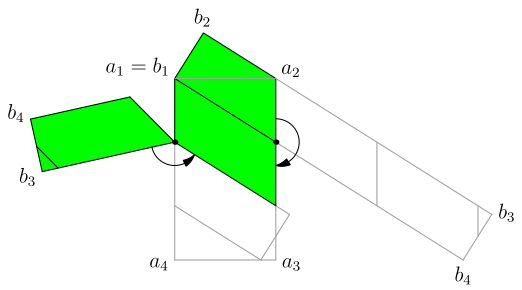

(a) Swinging $B$ back and forth to cover $A$.

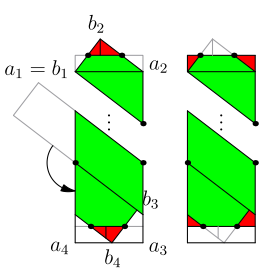

(b) Capping the top and bottom of $B$.

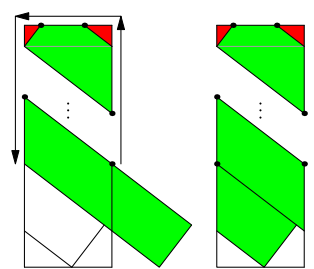

(c) Moving the end of $B$ to "wrap around" $A$.

Fig. 13 The stages of the rectangle-to-rectangle transformation

Montucla [19, 35], which is "almost" hinged in that all but one of the pieces is easy to hinge, and then we make it hinged using our unaltered rooted subtree movement tool from Lemma 14.

First we define a notion of "replacement" that will enable us to use the construction in the context of a larger hinged dissection. Suppose $P$ is a piece of a tree-like hinged figure $H$, and that $P$ has one or two distinct vertices $p, q$ belonging to hinges. Thus we can write $H=((A, a) \vee(P, p), q) \vee(B, b)$ for (possibly empty) rooted subtrees $(A, a)$ and $(B, b)$. A replacement in $H$ of piece $P$ with another polygon $P^{\prime}$ is a hinged figure $H^{\prime}=\left((A, a) \vee\left(P^{\prime}, p^{\prime}\right), q^{\prime}\right) \vee(B, b)$ for arbitrarily chosen vertices $p^{\prime}, q^{\prime}$ of $P^{\prime}$. (Here, we implicitly map $q^{\prime}$ to a corresponding vertex of $(A, a) \vee\left(P^{\prime}, p^{\prime}\right)$. Also, note that if $p^{\prime}=q^{\prime}$, then this mapping is not unique.)

Lemma 16 Any two rectangles $A$ and $B$ of equal area have a common hinged dissection. Furthermore, given a tree-like hinged figure $H$ in which $A$ is a piece with at most two vertices belonging to hinges, and given a refinement $G \prec H$, there is a hinged figure $F$ with $F \prec G$ and $F \prec H^{\prime}$, where $H^{\prime}$ is the replacement in $H$ of $A$ with $B$.

Proof Suppose by symmetry that $B$ has the smallest minimum side length. We begin by aligning both rectangles with their shorter edges on the horizontal axis and their longer edges on the vertical axis. Label the vertices of $A$ and $B$ by $a_{i}$ and $b_{i}$, respectively, for $i \in\{1,2,3,4\}$ starting at the top left and continuing clockwise. Next we identify the two top-left vertices $a_{1}$ and $b_{1}$, and then rotate $B$ counterclockwise (about $b_{1}$ ) until its lowest vertex $b_{4}$ is horizontally aligned with the base of $A$. The result is that the two rectangles have equal horizontal cross-section from $a_{1}=b_{1}$ down to $b_{3}$.

Figure 13(a) illustrates the first sequence of cuts. First we cut $B$ along $A$ 's right edge $a_{2} a_{3}$, hinging at the bottom of the cut, and rotate the extended portion of $B$ clockwise by $180^{\circ}$ to cover a strip of $A$. Again we cut $B$, now along the left side $a_{1} a_{4}$, hinging at the bottom, and rotate it back in, covering another horizontal strip of $A$. We continue in this way until the remaining segment of $B$ extending past $A$ 's boundary is no longer enough to cover an entire horizontal strip.

Next we cap the top subtriangle $a_{1} b_{2} a_{2}$ of $B$ as follows. We apply the transformation of Lemma 15 (Fig. 12) to this portion of the figure, cutting horizontally at 


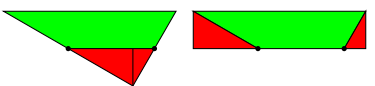

(a)

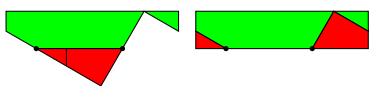

(b)

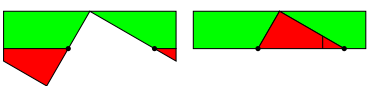

(c)

Fig. 14 The possible cases (up to reflection) when capping the rectangle base

half its height and vertically from $b_{2}$, then rotate the resulting pieces out to form a rectangular cap with the same width as $A$; see Fig. 13(b).

There are two cases on the bottom: the remaining segment of $B$ extends either up and left, or down and right. In the fortunate former case, we can perform the entire transformation using only classic-style manipulations, as shown in Fig. 13(b). First swing the extended portion back into $A$. This move results in a "triangular" bottom, similar to the triangle that was on top of $A$, but offset horizontally and wrapping through the edge of $A$. We can make this bottom rectangular by a transformation similar to the Lemma 15: cut horizontally at its vertical midpoint the same as before, and then vertically as shown in Fig. 14 so that the pieces line up with the border of $A$ when we swing them up. Every rectangle base (or its horizontal reflection) will fall into one of the three cases of Fig. 14, so this transformation is always possible.

Finally, we are left with the case where the end of $B$ extends down and to the right. Using our tools above, we can directly reduce this case to the previous case; refer to Fig. 13(c). First, we cut $B$ along edge $a_{2} a_{3}$, hinging at the top. To obtain a hinged dissection of $R$ and $R^{\prime}$, we can apply Lemma 5 to move the resulting subtree counterclockwise around the figure to line up with the left side of $A$ (as though it had wrapped around from the left side in the first place). This proves the first sentence of the lemma.

Now suppose that we have hinged figures $G \prec H$ where $H$ has $R$ as a piece with at most two vertices belonging to hinges. The construction in this case is identical except for the final rooted subtree movement in the last case. Let $\gamma$ be the counterclockwise boundary path of $R$ along which we wish to move the rooted subtree. If the hinge vertices of $R$ appear along $\gamma$, then the rooted subtree movement would follow the boundary of $H$ instead of $R$, and thereby modify pieces other than $R$, which we do not allow. To prevent this, we move any such hinge vertices out of the way first, by repeatedly applying unaltered rooted subtree movement of Lemma 14 to move the hinge farthest along $\gamma$ to the destination point of $\gamma$. Finally we can complete the desired movement of the subtree along $\gamma$, again by unaltered rooted subtree movement of Lemma 14.

\subsection{Pseudocuts}

Consider two hinged figures $G \prec F$. If we make a cut in $F$, producing $F^{\prime}$, we may not be able to make the same cut directly in $G$ : attempting to do so may disconnect the figure. In this section, we describe how to simulate such a cut in $G$, via an operation we call a pseudocut, which produces a hinged figure $H$ that is a refinement of both $G$ and $F^{\prime}$. This operation is essentially a more efficient version of iterated rooted subtree movement. 


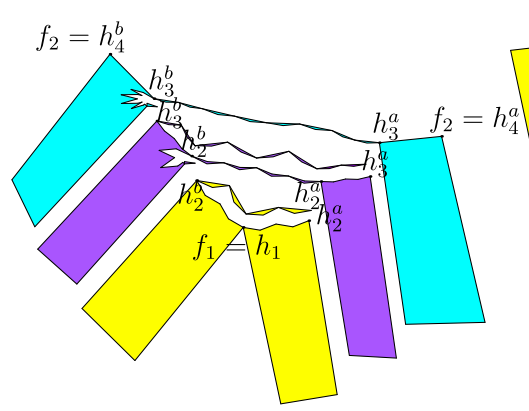

(a)

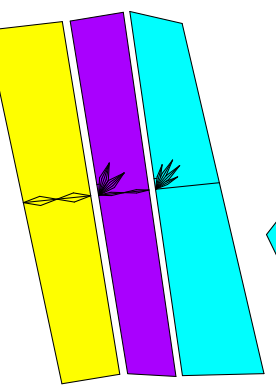

(b)

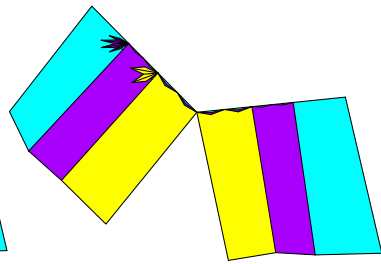

(c)

Fig. 15 Making a pseudocut across existing edges

Lemma 17 Let $f_{1}$ and $f_{2}$ be distinct boundary points of a common piece $P$ in a tree-like figure $F$, such that the segment $f_{1} f_{2}$ is interior to $P$ except at its endpoints. Let $F^{\prime} \prec F$ be the tree-like figure obtained by cutting $P$ along $f_{1} f_{2}$ and hinging at $f_{1}$. For any $g: G \prec F$, there is a common refinement $H$ with $H \prec G$ and $H \prec F^{\prime}$. Furthermore, $H$ differs from $G$ only within the pieces $P$ for which $g(P)$ intersects the relative interior of segment $f_{1} f_{2}$, and $H$ differs from $G$ only within the free regions incident to $f_{1} f_{2}$.

Proof Refer to Fig. 15. Let $h_{1}, h_{2}, \ldots, h_{n}$ be the points of intersection between the pseudocut $f_{1} f_{2}$ and the existing edges of $G$ as mapped by $g$. In particular, $h_{1}=f_{1}$ and $h_{n}=f_{2}$. After cutting along $f_{1} f_{2}$, distinguish identified vertices on each side as $h_{i}^{a}$ and $h_{i}^{b}$.

Our approach is to iteratively cut the pieces along $f_{1} f_{2}$, applying rooted subtree movement at each intersection point to maintain the needed connectivity. The key to the efficiency of this procedure is that, because each of these movements follows a nearly identical boundary path along the cut line, we can use approximately the same free regions and base vertices for each movement. This is similar to the behavior of the boundary chains in Lemma 5, where several chains use the same points, producing a series of kite chains nested within the outer triangle chains. More explicitly, we require that every movement over a previously used path includes all previously used base points, and that all additional base points interpolate between existing base points by even increments of inverse powers of 2 (that is, all base edges must arise by repeated halving of earlier edges). If we always select the smallest possible power of 2 (that is, the largest inverse power) that fits in the respective free regions, this will ensure that on overlapping path segments the length of each boundary chain is at most twice the length of the longest individual boundary chain, if we were to consider the movements independently.

We now proceed iteratively, applying the following procedure for each $i$ from 1 to $n-1$. (We assume $n>1$, for otherwise the whole operation is trivial.) Cut along the segment $h_{i} h_{i+1}$, hinging at $h_{i}$. In the case that there is a hinge at $h_{i+1}$ connecting to the current polygon, disconnect that hinge from $h_{i+1}^{b}$, leaving it connected at $h_{i+1}^{a}$. (In Fig. 15, this corresponds to disconnecting the "top" half of the cut from the hinge at $h_{i+1}$ so that it can pull away.) Now, if there is a hinge at $h_{i+1}$, it may connect 
to subtrees that need to be moved as well; that is, the cut and hinge in the original figure $F$ may induce a configuration that identifies these subtrees with $h_{i+1}^{b}$ rather than $h_{i+1}^{a}$. (In Fig. 15, this would correspond to a subtree that was entirely on the top side of the cut line.) If the hinge at $h_{i+1}$ connects to the current polygon, then we could have simply left these subtrees connected by a hinge to $h_{i+1}^{b}$ when we separated it. However, such a connection is not guaranteed, and in this case we will perform separate rooted subtree movements for each such subtree, moving it from $h_{i+1}^{a}$ to $h_{i+1}^{b}$.

In the end, we have cut the segment $f_{1} f_{2}$ by iteratively cutting the subsegments $h_{i} h_{i+1}$. By construction, the resulting figure is a refinement of $G$. Furthermore, if there were any additional hinge connections along the cut, we have performed rooted subtree movements to ensure that these connections trace around the boundary of the desired cut rather than crossing it, so that the resulting figure is also a refinement of $F^{\prime}$.

\subsection{Analysis}

We can now precisely specify the algorithm in terms of the various pieces we have developed. Intuitively, our procedure is the following.

1. Triangulate and chainify the polygons into two chains of triangles, each of area $\frac{1}{6}$ (Lemma 13).

2. Cut each triangle into three pieces and hinge into a rectangle (Lemma 15).

3. Apply the hinged dissection between matching pairs of rectangles (Lemma 16), combining with the existing dissection using pseudocuts (Lemma 17).

4. Use unaltered rooted subtree movement to reposition the hinges at the correct attachment points of each triangle (Lemma 14).

5. Apply Theorem 9 to the hinged dissection to guarantee a continuous motion between all of its configurations.

While these steps describe the conceptual breakdown of our hinged dissection, for efficiency we need to slightly modify the order. Specifically, pseudocuts are a relatively expensive operation, so we can only allow up to a constant number of them for each element in the chain. Thus, we cannot start with a triangle-to-rectangle hinged dissection and then overlay the whole rectangle-to-rectangle hinged dissection of Lemma 16 using pseudocuts, as it would use far more than a constant number of cuts in general. Instead, we actually start with chains of appropriately sized rectangles, and find a common hinged dissection between them using Lemma 16 first. Then we use pseudocuts to overlay the triangle-to-rectangle cuts of Lemma 15, of which there are only a constant number.

The actual algorithm that we analyze, then, is as follows.

1. Construct two chains of rectangles, each rectangle of area $\frac{1}{6}$, with dimensions matching those that would be obtained by sequentially applying Lemmas 13 and 15 to the input polygons.

2. Apply the hinged dissection between matching pairs of rectangles to obtain a single common hinged dissection for the two chains (Lemma 16). 
3. Using pseudocuts (Lemma 17), overlay this hinged dissection with triangle-torectangle hinged dissections (Lemma 15) to obtain (a refinement of) the original triangles produced by the triangulation and chainification as in the first step (Lemma 13).

4. Use unaltered rooted subtree movement to reposition the hinges at the correct attachment points of each triangle (Lemma 14).

5. Apply Theorem 9 to the hinged dissection to guarantee a continuous motion between all of its configurations.

Theorem 18 The preceding algorithm yields a hinged dissection between any pair of equal-area grid polygons, using a pseudopolynomial (that is, polynomial in the dimensions of the grid) number of pieces, in pseudopolynomial time.

Proof Let $n$ denote the total number of vertices among the two given polygons, and suppose that their vertices are drawn on an $N \times N$ integer grid (rational vertices can be handled by scaling). We have trivially that $n \leq N^{2}$. Because the triangulation and chainification splits the input polygons into chains of length $O\left(N^{2}\right)$, and the remainder of our construction works independently on each element of the chain, it suffices to show that our construction is pseudopolynomial (polynomial in $N$ ) when we apply Steps 2-4 to a single pair of rectangles generated by Step 1 . That this pseudopolynomial bound is then preserved by the post-processing in Step 5 follows immediately from the statement of Theorem 9.

We analyze the maximum number of pieces in our hinged dissection for a single pair of rectangles by considering the related question of the smallest possible nonzero value that can be computed at any intermediate step of the hinged dissection. This value gives a lower bound on, for instance, the smallest distance $\delta$ between any two distinct vertices. We can use this lower bound on $\delta$ to upper bound the number of pieces via a packing argument. Namely, every vertex is surrounded by an empty disk of radius $\delta$, so at most $N^{2} / \pi \delta^{2}$ such disks (and hence vertices) can fit in an area of $N^{2}$. The pieces in the figure form the faces of a planar graph with these vertices, so their number is maximized when the graph is a triangulation, resulting in an upper bound of $2 N^{2} / \pi \delta^{2}-4$ pieces. In particular, if $\delta \geq 1 / p(N)$ for some polynomial $p$, then the number of pieces is at most $2 N^{2} p(N)^{2} / \pi-4$, and is therefore polynomial.

Our task, then, is to show that the smallest nonzero value produced during the computation is indeed inverse pseudopolynomial, a problem that can be dealt with almost entirely algebraically. We use the root bounds of Burnikel et al. [4] which establish lower bounds on nonzero outcomes from algebraic computations viewed as a directed acyclic graph involving addition, subtraction, multiplication, division, and algebraic extensions (roots). Under these bounds, it suffices to show that the depth of the computation graph is bounded by a constant and that all arithmetic constants used by the computation are themselves pseudopolynomial (meaning they are rational values whose numerator and denominator are both bounded by a polynomial in $N$ ). Note that this is not the same as saying that the output vertices must be rational: irrational values are permitted as long as they arise from constant-degree algebraic extensions combined with constant-depth arithmetic computations.

We describe below how to compute the coordinates of all vertices of our figure with constant algebraic depth. This requires us to specify the construction more 
carefully, exploiting the inherent flexibility in how we choose many of the cuts. For each of the major operations in our construction-unaltered subtree movement, rectangle-to-rectangle hinged dissection, triangle-to-rectangle hinged dissection, and pseudocuts-we show that performing the operation increases the existing algebraic depth by at most an additive constant. The overall conclusion then follows by showing that each of these operations can occur at most a constant number of times for each rectangle pair in the original chains.

\subsubsection{Rotations and Kite Sweeps}

To maintain constant algebraic depth in our hinged dissections, it is important that we never rotate the same point or vector more than a constant number of (nested) times. For instance, we cannot have adjacent kites touching along a common angle, as we have described so far for simplicity, because then computing the coordinates of consecutive kites would require nested rotation. Instead, whenever we need to perform a rotation, we will use a rational approximation of the angle. We can approximate an angle within an arbitrary error bound $\epsilon$ by using a ratio of integers that are polynomial in $1 / \epsilon$ (for instance, by solving for an appropriate Pythagorean triple). We can further ensure that we always have plenty of room for such approximations, by restricting ourselves to free regions that use only half of the true available angular space, so we know that there will also be polynomial error tolerance built into any desired angles, and any single rotation will still involve only polynomial values. Kite sweeps will thus be spread out near each other but with angles ensuring that they do not intersect.

The preceding modification as described can produce nonsimple pieces, so we must also cut out small corners as in Step 3 of Lemma 5. The outermost small corner (the one described in Lemma 5) can be selected to have any convenient vertices that are simple rational computations with respect to the corresponding edges. (Any rational interpolation that does not intersect existing edges will work, so we can select one that does not increase the existing algebraic complexity by more than an additive constant.) But any required inner small corners can be produced without any additional computation at all: by the definition of a free region, we are guaranteed that there will be no other vertices or edges between adjacent kites. Therefore, we will re-use the central vertices of those kites, cutting edges between adjacent kites and hinging arbitrarily so that we preserve simplicity of the figure's polygons without adding any additional vertices.

\subsubsection{Computing Initial Rectangle Chains}

As the first step in the algorithm, we need to transform the input polygons into the necessary rectangle chains. It is straightforward to do this in constant depth, following Lemma 13. First we triangulate the vertices of the input polygons together with all grid points inside (inclusive). Clearly every resulting vertex will be pseudopolynomially bounded by definition: it will simply be some integer point contained in the input polygon. We then chainify as in Lemma 13, with each added vertex the average of three integer vertices, and thus at constant depth. 
To obtain the vertices of the rectangle chain from these computations, for each triangle we select the longest edge as the "base" to use in Lemma 15. (Comparing edge lengths does not affect our algebraic depth because the numerical results are not used elsewhere in the computation graph.) Then we compute the hinge points by taking the average of the respective base point with the apex. Next we compute the interior cut vertex by projecting the apex of the triangle down to the nearest point on the edge defined by the two hinges, a standard linear-algebra procedure. Finally, we obtain the remaining rectangle vertices by rotating the central vertex $180^{\circ}$ around the hinges.

Note that, during this procedure, we computed the location of the hinge points on the output rectangles while using only constant depth. We will use this fact again when we hinge-dissect back into triangles in Step 3.

\subsubsection{Unaltered Rooted Subtree Movements}

Unaltered rooted subtree movements occur in several contexts: during the rectangleto-rectangle transformation (up to three times, once for each of the two possible hinge points along the boundary path $\gamma$ and once for the final subtree movement to wrap around the rectangle), before each of the four pseudocuts for the triangle-to-rectangle transformation (up to two times per pseudocut), and finally up to four times in Step 4 when we relocate the connections to lie on the proper points at the base of each triangle (up to twice per triangle). There are thus at most 15 unaltered rooted subtree movements for each triangle in the overall chain, and we must show that any one of these increases the algebraic depth by at most an additive constant.

First, the free regions of the vertices and edges traversed by the boundary path are all simple computations with respect to the existing vertices as described in Sect. 3.4.1, though as in Sect. 5.7.1, we use rational approximations to the exact margins to avoid increasing depth.

Given these approximations to the free regions, the base vertices of the triangle chains cut out of the border of the figure can be computed by interpolating between the existing vertices by fractions, so that each base vertex is dependent only on the two adjacent boundary vertices and a rational fraction, the magnitude of which is within a constant factor of the length of the respective edge divided by the corresponding free-region bounds computed above. Because the free-region computations require constant algebraic depth, the base points can also be computed with constant depth, since any single such vertex can be computed independently based only on the two nearest vertices and the free-region bound, along with the rational interpolation factor.

Having computed the base points, the corresponding internal vertex for each chain can then be computed from its two base vertices by first computing the center point between the two base points (requiring one coordinate-wise addition and division by 2 ), then offsetting orthogonally (requiring rotation by $90^{\circ}$ ) by a sufficiently small rational offset. This yields the inner vertex, while depending on only two (already constant-depth) vertices, combined with a rational offset that, as in the preceding, is bounded by the respective free regions and can thus be chosen inverse pseudopolynomial. 
Having computed the vertices of the boundary triangle chain, we can compute the source's kite-sweep coordinates by re-using the triangle chain coordinates: translate each boundary triangle pair, to the source point (merging them into a kite by rotating one of them by $180^{\circ}$ ), and rotate into the free region by an appropriate rational angle as in Sect. 5.7.1. Because the angles chosen in this way are required only to be nonoverlapping, each kite location can be computed independently, and each individual vertex computation is still only a constant number of arithmetic operations based on the previous vertices, so constant depth is preserved. We maintain simplicity of the resulting pieces by cutting out the additional small corners described in Sect. 5.7.1.

\subsubsection{Rectangle to Rectangle}

Consider Step 2 of our algorithm, the rectangle-to-rectangle transformation. The longer dimension of either rectangle is at most $\sqrt{2} N$ because the entire figure was initially drawn on an $N \times N$ grid. Because by construction the area of each rectangle is $\frac{1}{6}$, this implies that the smaller dimension must be at least $\frac{1}{6} /(\sqrt{2} N)$.

Now we trace the transformation of Sect. 5.5, broken down into four substeps.

Step 1: Rotate the rectangles to their initial position. When we rotate the thicker rectangle to lie vertically, and rotate the narrower rectangle to have the same horizontal cross-section as the first, we require two quadratic extensions (square roots) to compute the rotation operation, as well as a constant number of simple arithmetic operations to actually perform the respective rotation on each vertex of both rectangles.

Step 2: Snake the longer rectangle across the shorter one in strips. Snaking the second rectangle back and forth along the first (Fig. 13(a)) can be done using only additions and subtractions, because all rotations are by $180^{\circ}$. However, chaining these operations by iteratively computing each strip from the preceding one would lead to nonconstant depth. Instead, we can compute the coordinates of the descending rectangle strips by observing that each strip appears at a fixed vertical distance from the preceding one. Thus, having computed that fixed distance once by intersecting the lower diagonal of rectangle $B$ with the right side of $A$, we can multiply it by increasing integers to find the vertex offset for each strip. These computations require a single (nonnested) multiplication for each additional vertex, combined with a vector addition to center the strip offset at the top vertices of $A$. By the dimension bounds above, the total number of strips is at most $\frac{\sqrt{2} N}{\frac{1}{6} /(\sqrt{2} N)}=12 N^{2}$, and thus any integer coefficient used in this step is still polynomially bounded, as required. Constant depth for the initial strips is thus maintained.

Step 3: Cap the triangles at each end of the rectangle. Capping the triangles at the top and bottom (Fig. 13(b)) requires rotations by $180^{\circ}$ and finding the midpoints of the triangles' altitudes, which is just a division by 2 . Thus this part of the computation requires only a constant number of operations in total.

Step 4: Relocate B's extra subtree back to the left of A, if needed. This step consists of up to three unaltered rooted subtree movements, which preserve constant depth as described in Sect. 5.7.3. 


\subsubsection{Pseudocuts}

As in the rectangle-to-rectangle transformation, performing a pseudocut may involve performing unaltered subtree movement on up to two external connecting hinges. As before, the efficiency of these operations follows directly from Sect. 5.7.3, so we need only consider the remainder of the operation.

Consider a single pseudocut whose defining points $f_{1}, f_{2}$ are at constant algebraic depth with respect to the other vertices in the configuration; this is the case in our transformation as shown in Sect. 5.7.2. When performing the pseudocut, we add vertices at every intersection of the cut line with an existing edge. Each such intersection point can be computed independently of the rest, and requires only a constant number of arithmetic operations, while depending only on the equations of the cut line and its intersecting edge, thus adding only constant depth to existing computations.

Having computed these intersection points, it only remains to add the chains and sweeps along the boundary of the cut. This stage is computed nearly identically to the unaltered rooted subtree movement in Sect. 5.7.3 above, but with additional constraints from the nesting of the chains. As in that case, we can obtain simple pseudopolynomial approximations to the free regions in this neighborhood. We can again compute the base vertices of the chain as simple rational interpolations of the respective edge's vertices. If we were to consider any single such movement in isolation, the interpolation coefficients of the base points would be pseudopolynomially bounded just as before. We are able to preserve this bound even in the presence of nested chains because of our rule about re-using base points in subsequent chains: all base points are still computed only from the original boundary vertices, and we need only alter the previous interpolation coefficients by multiplying by rational fractions whose denominator is (the smallest possible) power of 2 . This rule may be locally "wasteful" in the sense that all chains may grow to the length of the longest single chain, and may also be longer than necessary by as much as a factor of 2 , but by preserving the constant computation depth it gives us a better global guarantee.

The internal vertices of the chains are again computed as orthogonal offsets from the center of the respective base, and thus depend only on those two base vertices and an offset coefficient derived from the worst-case free-region bound. Because in this case successive chains will actually be nested inside each other, there may need to be up to polynomially many such offset coefficients, but if so they can be polynomially decreased as the chains are nested deeper. Again, however, each coefficient is selected as an independent approximation (rather than being computed algebraically from the previous offset).

The sweeps are again produced as a constant-depth computation derived from the boundary chain vertices, by first translating to the appropriate vertex, rotating one half $180^{\circ}$ into a triangle, and then rotating the whole triangle by an appropriate rational angle as described in Sect. 5.7.1. Each such triangle is again independent of the others: every target vertex depends only on its source triangle, its destination vertex, and its computed (nonoverlapping) rational rotation. Additional small corners to cut out to preserve simplicity of the pieces are again generated as in Sect. 5.7.1, preserving constant depth. 


\subsubsection{Triangle to Rectangle}

After mapping between the two rectangles, we need to ensure that our hinged dissection can also transform into both of the original area- $\frac{1}{6}$ triangles via the simple triangle-to-rectangle hinged dissection of Lemma 15. Each triangle-to-rectangle hinged dissection requires two cuts, and we need to perform two of these transformations, one for each target triangle. We add the required four additional cuts using our pseudocut construction from Lemma 17. We know from Sect. 5.7.2 that the cut lines for the pseudocut have coordinates that are pseudopolynomially bounded with respect to the existing computation graph. Then Sect. 5.7.5 above implies that each pseudocut adds only constant depth to any of the algebraic computations. Because we need to make only four such cuts, this stage also adds only constant depth.

\subsubsection{The End}

The preceding sections show that the computations at each stage of the hinged dissection can be performed with only constant algebraic depth for each triangle/rectangle pair. It follows that each such pair is cut into at most a pseudopolynomial number of additional pieces, implying the global pseudopolynomial bound as required. Because furthermore all the described computation steps are constructive, the hinged dissection can be performed algorithmically in pseudopolynomial time.

This concludes the proof of Theorem 18.

\section{Higher Dimensions}

We now briefly discuss hinged figures in three dimensions. A $3 D$ hinged figure is a collection of polyhedra called pieces hinged along common positive-length edges called hinges. As before, the cyclic order of pieces around a hinge must remain fixed.

Not every two polyhedra of equal volume have a common dissection. Dehn [11] proved an invariant that must necessarily match between the two polyhedra. For example, Dehn's invariant forbids any two distinct Platonic solids from having a common dissection. Many years later, Sydler [39] proved that polyhedra $A$ and $B$ have a common dissection if and only if $A$ and $B$ have the same volume and the same Dehn invariant. Jessen [25] simplified this proof by an algebraic technique and generalized the result to 4D polyhedral solids. (The 5D and higher cases remain open.) Dupont and Sah [17] gave another proof that illustrates further connections to algebraic structures. Kreinovich [28] show that Dehn's condition is decidable.

Clearly, if two polyhedra have no common dissection, then they also have no common hinged dissection. This presents the following natural conjecture:

Conjecture 19 Given $n$ polyhedra $P_{1}, P_{2}, \ldots, P_{n}$ of equal volume and equal Dehn invariant, there exists a $3 D$ hinged figure $H$ with $H \prec P_{i}$ for $1 \leq i \leq n$.

We believe that methods similar to those used in this paper can be used to prove Conjecture 19. With some more care, we also believe that it is possible to guarantee non-self-intersecting motions in this extension. Finally, we conjecture that our 
techniques generalize further to refining dissections of polyhedral solids in arbitrary dimensions into equivalent hinged dissections.

Acknowledgements This work arose during a series of open-problem sessions for an MIT class on Geometric Folding Algorithms (6.885 in Fall 2007). We thank the other participants of those sessions for providing a productive and inspiring environment. We also thank Stefan Langerman for helpful discussions. Finally, we are grateful to referees Joseph O'Rourke and Joseph Mitchell, and two anonymous referees, for many helpful comments on the paper.

\section{References}

1. Abbott, T.G., Demaine, E.D., Gassend, B.: A generalized Carpenter's Rule Theorem for self-touching linkages, January 2009. arXiv:0901.1322

2. Akiyama, J., Nakamura, G.: Dudeney dissection of polygons. In: Revised Papers from the Japan Conference on Discrete and Computational Geometry, Tokyo, Japan. Lecture Notes in Computer Science, vol. 1763, pp. 14-29. Springer, Berlin (1998)

3. Akiyama, J., Nakamura, G., Nozaki, A., Ozawa, K., Sakai, T.: The optimality of a certain purely recursive dissection for a sequentially $n$-divisible square. Comput. Geom., Theory Appl. 24(1), 27-39 (2003)

4. Burnikel, C., Funke, S., Mehlhorn, K., Schirra, S., Schmitt, S.: A separation bound for real algebraic expressions. In: Proceedings of the 9th Annual European Symposium on Algorithms, Aarhus, Denmark. Lecture Notes in Computer Science, vol. 2161, pp. 254-265. Springer, Berlin (2001)

5. Bolyai, F.: Tentamen juventutem studiosam in elementa matheseos purae, elementaris ac sublimioris, methodo intuitiva, evidentiaque huic propria, introducendi. Typis Collegii Refomatorum per Josephum et Simeonem Kali, Maros Vásárhely (1832-1833)

6. Connelly, R., Demaine, E.D., Rote, G.: Straightening polygonal arcs and convexifying polygonal cycles. Discrete Comput. Geom. 30(2), 205-239 (2003)

7. Connelly, R., Demaine, E.D., Demaine, M.L., Fekete, S., Langerman, S., Mitchell, J.S.B., Ribó, A., Rote, G.: Locked and unlocked chains of planar shapes. In: Proceedings of the 22nd Annual ACM Symposium on Computational Geometry, Sedona, Arizona, pp. 61-70 (2006)

8. Cohn, M.J.: Economical triangle-square dissection. Geom. Dedic. 3, 447-467 (1975)

9. Czyzowicz, J., Kranakis, E., Urrutia, J.: Dissections, cuts, and triangulations. In: Proceedings of the 11th Canadian Conference on Computational Geometry, Vancouver, Canada (1999). http://www.cs.ubc.ca/conferences/CCCG/elec_proc/c33.ps.gz

10. Davis, J.F., Kirk, P.: Lecture Notes in Algebraic Topology. American Mathematical Society, Providence (2001)

11. Dehn, M.: Über den Rauminhalt. Nachrichten von der Gesellschaft der Wissenschaften zu Göttingen, Mathematisch-Physikalische Klasse, pp. 345-354 (1900). Later published in Math. Ann. 55, 465-478 (1902)

12. Demaine, E.D., Eppstein, D., Erickson, J., Hart, G.W., O'Rourke, J.: Vertex-unfolding of simplicial manifolds. In: Discrete Geometry: In Honor of W. Kuperberg's 60th Birthday, pp. 215-228. Dekker, New York (2003)

13. Demaine, E.D., Mitchell, J.S.B., O'Rourke, J.: Problem 47: Hinged dissections. In The Open Problems Project, March 2003. http://www.cs.smith.edu/ orourke/TOPP/P47.html

14. Demaine, E.D., Demaine, M.L., Eppstein, D., Frederickson, G.N., Friedman, E.: Hinged dissection of polyominoes and polyforms. Comput. Geom., Theory Appl. 31(3), 237-262 (2005)

15. Demaine, E.D., Demaine, M.L., Lindy, J.F., Souvaine, D.L.: Hinged dissection of polypolyhedra. In: Proceedings of the 9th Workshop on Algorithms and Data Structures, Waterloo, Canada. Lecture Notes in Computer Science, vol. 3608, pp. 205-217 (2005)

16. Dudeney, H.E.: Puzzles and prizes. Weekly Dispatch, 1902. The puzzle appeared in the April 6 issue of this column. An unusual discussion followed on April 20, and the solution appeared on May 4

17. Dupont, J.L., Sah, C.-H.: Homology of Euclidean groups of motions made discrete and Euclidean scissors congruences. Acta Math. 164(1), 1-27 (1990)

18. Eppstein, D.: Hinged kite mirror dissection, June 2001. arXiv:cs.CG/0106032

19. Frederickson, G.N.: Dissections: Plane and Fancy. Cambridge University Press, Cambridge (1997) 
20. Frederickson, G.N.: Hinged Dissections: Swinging \& Twisting. Cambridge University Press, Cambridge (2002)

21. Gerwien, P.: Zerschneidung jeder beliebigen Anzahl von gleichen geradlinigen Figuren in dieselben Stücke. J. Reine Angew. Math. (Crelle's J.) 10, 228-234 (1833) and Taf. III

22. Griffith, S.: Growing Machines. PhD thesis, Media Laboratory, Massachusetts Institute of Technology, September 2004

23. Grünbaum, B., Shephard, G.C.: Pick's theorem. Am. Math. Mon. 100(2), 150-161 (1993)

24. Hatcher, A.: Algebraic Topology. Cambridge University Press, Cambridge (2002)

25. Jessen, B.: The algebra of polyhedra and the Dehn-Sydler theorem. Math. Scand. 22, 241-256 (1968)

26. Kelland, P.: On superposition, part II. Trans. R. Soc. Edinb. 33, 471-473 (1864) and plate XX

27. Kranakis, E., Krizanc, D., Urrutia, J.: Efficient regular polygon dissections. Geom. Dedic. 80, $247-$ $262(2000)$

28. Kreinovich, V.: Equidecomposability (scissors congruence) of polyhedra in $\mathbb{R}^{3}$ and $\mathbb{R}^{4}$ is algorithmically decidable: Hilbert's 3rd problem revisited. Geombinatorics 18(1), 26-34 (2008)

29. Lemon, D.: The Illustrated Book of Puzzles. Saxon, London (1890)

30. Lindgren, H.: Recreational Problems in Geometric Dissections and How to Solve Them. Dover, New York (1972). Revised and enlarged by Greg Frederickson

31. Lowry, M.: Solution to question 269, [proposed] by Mr. W. Wallace. In: Leybourn, T. (ed.) Mathematical Repository, part 1, vol. 3, pp. 44-46. W. Glendinning, London (1814)

32. Madachy, J.S.: Geometric dissections. In: Madachy's Mathematical Recreations, pp. 15-33. Dover, New York (1979), Chap. 1. Reprint of Mathematics on Vacation, Scribner, 1975

33. Mao, C., Thallidi, V.R., Wolfe, D.B., Whitesides, S., Whitesides, G.M.: Dissections: Self-assembled aggregates that spontaneously reconfigure their structures when their environment changes. J. Am. Chem. Soc. 124, 14508-14509 (2002)

34. O'Rourke, J.: Computational geometry column 44. Int. J. Comput. Geom. Appl. 13(3), 273-275 (2002)

35. Ozanam, J.: Récréations Mathématiques et Physiques, pp. 297-302. Claude Antoine Jombert, fils, Paris (1778). According to [19], these pages were added to the book by Jean Montucla under the pseudonym M. de Chanla

36. Panckoucke, A.-J.: Les Amusements Mathématiques. Chez André-Joseph Panckoucke, Lille (1749)

37. Pick, G.: Geometrisches zur Zahlenlehre. Sitzungsber. Dtsch. Naturwissenschaftlich-Medicinischen Ver. Böhmen "Lotos" Prag 19, 311-319 (1900)

38. Rus, D., Butler, Z., Kotay, K., Vona, M.: Self-reconfiguring robots. Commun. ACM 45(3), 39-45 (2002)

39. Sydler, J.-P.: Conditions nécessaires et suffisantes pour l'équivalence des polyèdres de l'espace euclidien à trois dimensions. Comment. Math. Helv. 40, 43-80 (1965)

40. Wallace, W. (ed.): Elements of Geometry, 8th edn. Bell \& Bradfute, Edinburgh (1831) 